\title{
Systematic analysis identifies three-IncRNA signature as a potentially prognostic biomarker for lung squamous cell carcinoma using bioinformatics strategy
}

\author{
Jing $\mathrm{Hu}^{1,2 *}$, Lutong $\mathrm{Xu}^{3 \#}$, Tao Shou ${ }^{1,2}$, Qiang $\mathrm{Chen}^{4}$ \\ ${ }^{1}$ Department of Medical Oncology, The First People's Hospital of Yunnan Province, Kunming 650032, China; ${ }^{2}$ Department of Medical Oncology, \\ The Affiliated Hospital of Kunming University of Science and Technology, Kunming 650032, China; ${ }^{3}$ Faculty of Life Science and Technology, \\ Kunming University of Science and Technology, Kunming 650500, China; ${ }^{4}$ Faculty of Animal Science and Technology, Yunnan Agricultural \\ University, Kunming 650201, China \\ Contributions: (I) Conception and design: Q Chen, T Shou; (II) Administrative support: Q Chen, T Shou; (III) Provision of study materials or \\ patients: J Hu, L Xu; (IV) Collection and assembly of data: J Hu, L Xu, Q Chen; (V) Data analysis and interpretation: J Hu, L Xu; (VI) Manuscript \\ writing: All authors; (VII) Final approval of manuscript: All authors. \\ "These authors contributed equally to this work. \\ Correspondence to: Qiang Chen. Faculty of Animal Science and Technology, Yunnan Agricultural University, No. 95 of Jinhei Road, Kunming 650201, \\ China. Email: chq@sjtu.edu.cn; Tao Shou. Department of Medical Oncology, The First People’s Hospital of Yunnan Province, No. 157 of Jinbi \\ Road, Kunming 650032, China. Email: taoshou_yn@163.com.
}

Background: Lung squamous cell carcinoma (LUSC) is the second most common histological subtype of lung cancer (LC), and the prognoses of most LUSC patients are so far still very poor. The present study aimed at integrating lncRNA, miRNA and mRNA expression data to identify lncRNA signature in competitive endogenous RNA (ceRNA) network as a potentially prognostic biomarker for LUSC patients.

Methods: Gene expression data and clinical characteristics of LUSC patients were retrieved from The Cancer Genome Atlas (TCGA) database, and were integratedly analyzed using bioinformatics methods including Differentially Expressed Gene Analysis (DEGA), Weighted Gene Co-expression Network Analysis (WGCNA), Protein and Protein Interaction (PPI) network analysis and ceRNA network construction. Subsequently, univariate and multivariate Cox regression analyses of differentially expressed lncRNAs (DElncRNAs) in ceRNA network were performed to predict the overall survival (OS) in LUSC patients. Receiver operating characteristic (ROC) analysis was used to evaluate the performance of multivariate Cox regression model. Gene expression profiling interactive analysis (GEPIA) was used to validate key genes.

Results: WGCNA showed that turquoise module including 1,694 DElncRNAs, 2,654 DEmRNAs as well as 113 DEmiRNAs was identified as the most significant modules (cor=0.99, $\mathrm{P}<1 \mathrm{e}-200$ ), and differentially expressed RNAs in the module were used to subsequently analyze. PPI network analysis identified FPR2, GNG11 and ADCY4 as critical genes in LUSC, and survival analysis revealed that low mRNA expression of FPR2 and GNG11 resulted in a higher OS rate of LUSC patients. A lncRNA-miRNA-mRNA ceRNA network including 121 DElncRNAs, 18 DEmiRNAs and 3 DEmRNAs was established, and univariate and multivariate Cox regression analysis of those 121 DElncRNAs showed a group of 3 DElncRNAs (TTTY16, POU6F2-AS2 and CACNA2D3-AS1) had significantly prognostic value in OS of LUSC patients. ROC analysis showed that the area under the curve (AUC) of the 3-lncRNA signature associated with 3-year survival was 0.629 .

Conclusions: The current study provides novel insights into the lncRNA-related regulatory mechanisms underlying LUSC, and identifying 3-lncRNA signature may serve as a potentially prognostic biomarker in predicting the OS of LUSC patients.

Keywords: Lung squamous cell carcinoma (LUSC); weighted gene co-expression network analysis (WGCNA); competing endogenous RNA network; long non-coding RNA; overall survival (OS) 
Submitted Apr 10, 2019. Accepted for publication Aug 29, 2019.

doi: 10.21037/tlcr.2019.09.13

View this article at: http://dx.doi.org/10.21037/tlcr.2019.09.13

\section{Introduction}

Lung cancer (LC) with more than 2 million new cases and 1.7 million deaths in 2018 , is the most commonly diagnosed malignancies and the top leading cause of cancer death worldwide (1). Non-small cell lung cancer (NSCLC) is the most common pathological type, and accounts for approximately $85 \%$ of all LCs (2). Among NSCLCs, lung squamous cell carcinoma (LUSC) with more than 400,000 new cases per year is the second most common type of NSCLCs, and accounts for $20-30 \%$ of NSCLCs $(3,4)$. Due to the lack of sensibility to radiotherapy, surgery and chemotherapy are the main methods to combat LUSC (5). Despite advances in treatment methods of LUSC, the 5 -year overall survival (OS) rate of LUSC patients with clinical I and II stages is about $40 \%$, and that of LUSC patients with clinical III and IV stages less than poor 5\% (6). A diagnosis for LUSC at an early stage contributes to offering a favorable prognosis, and the 5-year OS rate will significantly increase to $70-90 \%$ (7). Currently, disease stage and histological grade are still the basic methods for evaluating the diagnosis and prognosis of LUSC. However, it is limited to evaluate the predictive value in diagnosing early-stage LUSC by clinical and pathological symptoms, and it is difficult to predict the clinical outcomes due to the heterogeneity of LUSC. In addition, the molecular mechanisms underlying LUSC still remain unclear. Therefore, it is vital to further explore the molecular mechanisms, and identify potentially molecular diagnostic and prognostic markers and/or therapeutic targets to combat LUSC.

Smoking is the major risk factor for LUSC, which causes high number of mutations such as TP53, CDKN $2 A$ and PIK3CA (8). Although molecularly targeted therapies directly against those driver genes are an effective method to combat LUSC, development of molecularly targeted therapies has been less rapid for LUSC with respect to lung adenocarcinoma (LUAD), and molecularly targeted therapies are still not standard among LUSCs. Even though some genes such as DDR2, AKT and FGFR were considered as potentially molecular targets, few effective targeted therapies for LUSC are so far approved, and targeted therapies developed against LUAD are largely ineffective against LUSC $(9,10)$. Recent efforts have focused on discovering novel, clinically actionable cancer driver genes by multi-omics analysis $(10,11)$. However, due to the accumulation of large number of passenger mutations from prolonged exposure to carcinogens, identifying novel driver genes is challenging (8).

Long non-coding RNAs (lncRNAs) are an important class of non-coding RNAs (ncRNAs) with over 200 nucleotides long (12), and are widely reported to play roles as important regulatory components in tumor initiation and progression (13). However, little knowledge about the roles of lncRNAs is known in tumor biology. Nevertheless, due to the high tissue specificity of lncRNA expression, lncRNAs have been considered as potentially valuable diagnostic and prognostic biomarkers and/or therapeutic targets of some malignant tumors (14-17). Competitive endogenous RNA (ceRNA) hypothesis is so far considered as the foundation of playing the regulatory role for lncRNAs (18). LncRNA as ceRNA and mRNA can competitively bind microRNA (miRNA) by miRNA-response-elements (MREs) to play key roles in various biological processes such as tumor formation and metastasis (19). For instance, Fan's team constructed lncRNA-miRNA-mRNA ceRNA network based on ceRNA theory to identify that a 4-lncRNA signature played roles in breast cancer (20). Liu and the colleagues revealed functional lncRNAs in gastric cancer by integrative analysis of dysregulated lncRNA-related ceRNA network (21). In addition, systematic analysis of lncRNA-related ceRNA network have been reported in many other cancers including thyroid carcinoma (22), glioblastoma (23), prostate cancer (24), and ovarian cancer (25). Collectively, the lncRNA-related ceRNA network findings revealed the lncRNA-associated regulatory mechanisms by miRNA-mediated lncRNA and mRNA interaction in cancer initiation and progression. For lncRNA-related ceRNA network of LUSC, several studies have reported some lncRNAs associated with LUSC development and clinical outcome $(9,26,27)$. However, the findings reported from different studies are inconsistent due to differentially limited samples. The Cancer Genome Atlas (TCGA, 2018, the seventh edition of TNM staging criteria, https://cancergenome.nih.gov/) is a publically available cancer-related database which provides large- 
scale multi-dimensional molecular profile data related to over 30 cancer types. Using LUSC-related data with large sample size from TCGA database to identify LUSC-specific IncRNAs can increase statistical reliability of the current study. Another key issue is that most reported studies didn't consider the co-expression characteristic of genes in ceRNA network, which may be difficult to target the key lncRNAs. Weighted gene co-expression network analysis (WGCNA) is a systematic biology method to describe the correlation patterns among genes across samples, and using WGCNA can find modules of highly correlated genes and identify key genes (28). Presently, WGCNA has been successfully applied in various biological contexts including cancer such as LC (29), bladder cancer (30), and colon cancer (31). Systematic analysis of lncRNA-miRNA-mRNA ceRNA network based on WGCNA contributes to insight into novel molecular mechanisms involved in LUSC. While, very little information is available on LUSC ceRNA based on WGCNA.

In this work, LUSC-related RNA-seq data were retrieved from the TCGA database, and were systematically integrated and analyzed based on bioinformatics methods including Differentially Expressed Gene Analysis (DEGA), WGCNA, Protein and Protein Interaction (PPI) network, Gene Ontology (GO) and Kyoto Encyclopedia of Genes and Genomes (KEGG) pathway analyses, ceRNA network, and centrality analysis. Subsequently, survival analysis was performed to identify the key lncRNA signature in lncRNA-related ceRNA network as potentially valuable prognosticator associated with OS of LUSC patients. This study provides novel insights into molecular mechanisms underlying LUSC, and identifying novel lncRNAs may serve as potentially diagnostic and prognostic biomarkers and/or therapeutic targets to combat LUSC.

\section{Methods}

The flow chart of systematic bioinformatics analysis in the current study was showed in Figure 1.

\section{RNA-seq data and patient information collection}

LUSC-related RNA-seq data were retrieved from TCGA database portal (2018, https://cancergenome.nih.gov/), and the inclusion criteria of RNA-seq data were included as follows: (I) histological diagnosis for LUSC; (II) except LUSC no other malignancy; (III) data with complete clinical information. A total of 475 LUSC tissues and
38 non-LUSC normal lung tissues RNA-seq data were retrieved. Among, 38 non-LUSC normal lung tissues were from the normal lung tissues nearby LUSC tissues of 38 LUSC patients. The clinical information of all LUSC samples patients was simultaneously obtained from TCGA database. Sample clustering was used to detect outlier, and 2 LUSC tissues samples were excluded. Finally, a total 473 LUSC-related and 38 non-LUSC normal lung tissues RNA-seq data was included. All RNA-seq data were approved by the Institutional Review Board of the relevant participating institutions and by the National Cancer Institute of NIH. No approval from the ethics committee was required. The present study meets the requirements of data usage and publishing from TCGA database. The clinical characteristics for LUSC patients were listed in Table 1.

\section{RNA-seq data preprocessing and DEGA}

All raw RNA-seq data were subjected to normalization using the trimmed mean of M-values method.

Edge R package in Bioconductor project (version 3.8, http://www.bioconductor.org/) was used to screen the differentially expressed lncRNAs (DElncRNAs), differentially expressed miRNAs (DEmiRNAs) and differentially expressed mRNAs (DEmRNAs) between LUSC tissues and non-LUSC lung tissues samples (32). $\mid \log$ (fold change) $\mid(|\log \mathrm{FC}|)>2$ and statistical $\mathrm{P}$ value $<0.01(\mathrm{P}<0.01)$ were set as cut-off criteria. The volcano plots of RNA expression were visualized using the ggplot2 package (version 3.1.0, https://github.com/tidyverse/ ggplot2). The heat map of differentially expressed RNAs (DERNAs) was plotted using the pheatmap package (version 1.0.10, https://CRAN.R-project.org/package=pheatmap).

\section{$W G C N A$}

WGCNA was used to construct the gene co-expression network among DElncRNAs, DEmiRNAs and DEmRNAs, and identify co-expression gene modules. WGCNA package (version 1.13) based on $\mathrm{R}$ was used to perform WGCNA (28). The expression matrix was firstly converted into an adjacency matrix, and an unsupervised co-expression relationship was constructed based on the adjacency matrix using Pearson correlation coefficients for gene pairs. The correlation adjacency matrix was strengthened by power $\beta=10$ (soft threshold), and the power parameter was selected based on scale-free topology criterion. Secondly, the 


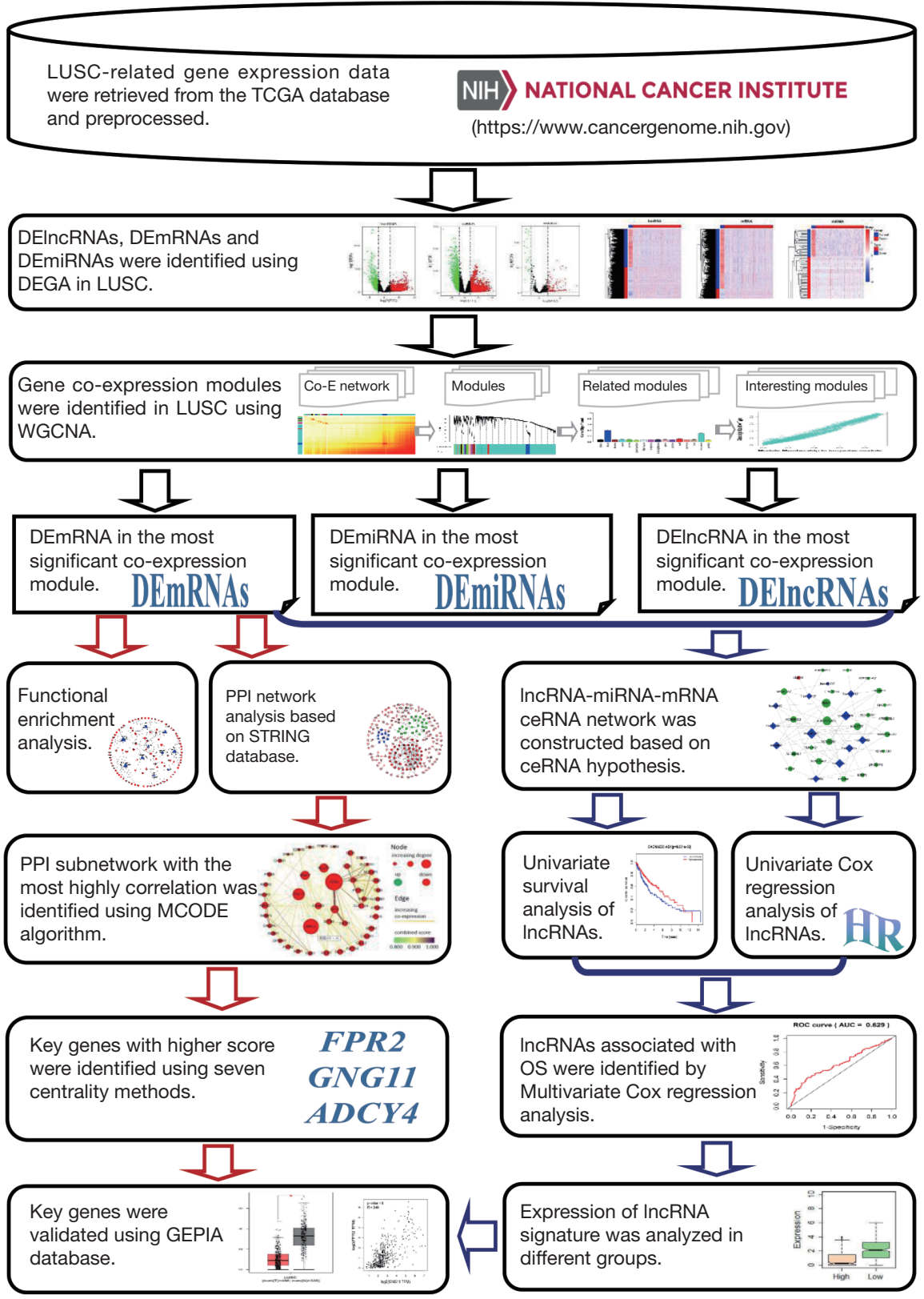

Figure 1 Flow chart of bioinformatics analysis. LUSC, lung squamous cell carcinoma; DEGA, differentially expressed gene analysis; DElncRNA, differentially expressed lncRNA; WGCNA, weighted gene co-expression network analysis; PPI, protein and protein interaction; ceRNA, competitive endogenous RNA; MCODE, molecular complex detection; GEPIA, gene expression profiling interactive analysis.

adjacency matrix was converted into a topological matrix, and topological overlap measure (TOM) was used to measure the correlation of gene pairs. According to TOMbased dissimilarity, average linkage hierarchical clustering was performed to classify genes with coherent expression profiles into gene modules. Dynamic cutting algorithm was used to identify gene modules from the system cluster tree, and the modules with $95 \%$ similarity were merged into a module. Module eigengene (ME) was defined as the first principal components, and was the representative in 
Table 1 The predictive values of clinical features and risk score

\begin{tabular}{lccccc}
\hline \multirow{2}{*}{ Variable } & Patients (N) & \multicolumn{2}{c}{ Univariate analysis } & & \multicolumn{2}{c}{ Multivariate analysis } \\
\cline { 3 - 4 } & & HR $(95 \% \mathrm{Cl})$ & $\mathrm{P}$ & $\mathrm{HR}(95 \% \mathrm{Cl})$ & $\mathrm{P}$ \\
\hline Gender (male/female) & $371 / 130$ & $0.955(0.694-1.316)$ & 0.780 & $1.095(0.793-1.512)$ & 0.580 \\
Pathologic stage (I-II/III-IV) & $406 / 95$ & $1.226(0.894-1.681)$ & 0.206 & $0.805(0.586-1.105)$ & 0.179 \\
Smoking (yes/no) & $222 / 279$ & $0.778(0.593-1.020)$ & 0.069 & $1.301(0.991-1.710)$ & 0.058 \\
\hline
\end{tabular}

$\mathrm{HR}$, hazard ratio; $\mathrm{Cl}$, confidence interval.

module genes. Module membership (MM) was defined as the correlation between ME and gene module. Gene significance (GS) was indexed by $\log 10$ transformation of the $\mathrm{p}$ value of $t$-test measuring differential expression between LUSC and non-LUSC tissues. Module significance (MS) was defined as the average GS for all the genes in the module. More detailed description of WGCNA is presented by original article (28).

The genes in the most significant module were selected to further analyze.

\section{PPI network construction and analysis}

The interactive relationships among differentially expressed genes (DEGs) encoding proteins in the most significant gene co-expression module was analyzed by constructing a PPI network. The interactive information among DEGs encoding proteins was retrieved from online STRING database (version 11.0, https://string-db.org/) (33). The gene pairs with a combined score $\geq$ the highest confidence 0.9 were used to construct the PPI network. Cytoscape software (version 3.7.0, http://www.cytoscape.org/) was used to construct and visualize the interactive relationships among genes in whole PPI network (34). The subnetwork (highly correlated module) was extracted from whole PPI network using Molecular COmplex DEtection (MCODE) algorithm based on topological properties of whole PPI network, and a plugin MCODE (version 1.5.1) in Cytoscape was used to perform MCODE analysis (35). The threshold parameters were more severely set for Degree Cutoff $=4$, Node Score Cutoff=0.6, K-Core $=4$ and Max. Depth $=100$. The most significantly correlated module was used to next analyze.

\section{Gene function analysis}

GO and KEGG pathway enrichment analyses of DEmRNAs in PPI network were implemented using the
clusterProfiler package (version 3.10.1), and an adjusted $\mathrm{P}<0.05$ was considered to significantly enrich (36). GO analysis included Biological Process (BP), Cellular Component (CC) and Molecular Function (MF).

\section{Identification of key genes and validation}

Key genes in PPI network were identified using Centrality analyses. Centrality analyses including Subgraph Centrality, Degree Centrality, Eigenvector Centrality, Betweenness Centrality, Network Centrality, Information Centrality and Closeness Centrality were performed using a plugin CytoNCA (version 2.1.6) in Cytoscape (37). The genes with higher Centrality scores were identified as key gene, and essential genes were identified as intersecting genes of key genes obtained by seven Centrality methods. Gene Expression Profiling and Interactive Analyses (GEPIA, http://gepia.cancer-pku.cn) database is an interactive web server for analyzing gene expression data of tumors and normal tissues from TCGA and genotype-tissue expression database $(38,39)$, and was used to validate the expression of essential genes in PPI network between LUSC and nonLUSC lung normal tissues and analyze the association with OS in LUSC patients. In GEPIA database, the expression analysis between LUSC and non-LUSC lung normal tissues was performed using $\log 2(\mathrm{TPM}+1)$, and survival analysis was perform using Log-Rank (LR) test (Mantel-Cox test)for hypothesis test. The LR P value, cox proportional hazard ratio (HR) and the $95 \%$ confidence interval (CI) information were calculated.

\section{Construction of lncRNA-miRNA-mRNA ceRNA network}

Co-expression genes in the most significant module were used to construct IncRNA-miRNA-mRNA ceRNA network, and ceRNA network was established based on ceRNA hypothesis that lncRNA directly bind miRNA by acting as miRNA sponge to indirectly regulate the 
function of mRNA (18). A ceRNA network was constructed according to the following steps: (I) three types of DERNAs in the most significant module were kept; (II) the potential target miRNAs of DElncRNAs, as well as lncRNAs and miRNAs interaction relationships were predicted using the online miRcode tool (miRcode 11, http://www.mircode. org/); (III) the potential target mRNAs of DEmiRNAs were predicted using the online tools including TargetScan (release 7.2, http://www.targetscan.org/), miRDB (version 5.0, http://mirdb.org/) and miRTarBase (release 7.0, http:// mirtarbase.mbc.nctu.edu.tw/); (IV) based on ceRNA hypothesis, intersecting miRNAs negatively regulated by lncRNAs and mRNAs were selected to construct ceRNA network. A ceRNA network was constructed and visualized using an open-source Cytoscape software (34).

\section{LUSC-specific prognostic lncRNA signatures identification}

The associations between DElncRNAs in ceRNA network and OS in LUSC patients were evaluated using KaplanMeier (KM) estimate and Log-rank (LR) test in survival package (version 2.43-3, https://CRAN.R-project.org/ package $=$ survival) based on R. Group cut off of $50 \%$ was set, and LR P value, hazard ratio (HR) and $95 \%$ CI were computed. The statistical $\mathrm{P}<0.05$ was considered as the significant association between DElncRNA and OS of LUSC patients. Further, univariate Cox proportional hazards regression model on the basis of survival package in $\mathrm{R}$ project was performed to evaluate the association between DElncRNAs in ceRNA network and OS of LUSC patients. The same characteristic parameters as LR method were computed and the same significant $\mathrm{P}$ value criterion was set. Overlapping DElncRNAs significantly associated with OS of LUSC patients in LR method and univariate Cox regression analysis was performed multivariate Cox hazards regression model with Stepwise method, and which was implemented using survival package in $\mathrm{R}$ project, and multivariate Cox hazards regression model was used to assess the prognostic value for LUSC. DElncRNA combination in the optimal Cox hazard regression model was used to further analyses. The hazards model was established as follows:

$$
\begin{aligned}
\text { Risk score } & =\operatorname{Exp}_{\operatorname{lncRNA} 1}{ }^{*} \operatorname{Coe}_{\operatorname{lncRNA1}}+\operatorname{Exp}_{\operatorname{lncRNA2} 2}{ }^{*} \operatorname{Coe}_{\operatorname{lncRNA} 2} \\
& +\operatorname{Exp}_{\operatorname{lncRNA} 3}{ }^{*} \operatorname{Coe}_{\operatorname{lncRNA} 3}+\ldots \operatorname{Exp}_{\operatorname{lncRNAn}}{ }^{*} \operatorname{Coe}_{\operatorname{lncRNAn}}
\end{aligned}
$$

where "Exp" denoted the expression level of lncRNA, and "Coe" represented the regression coefficient from the multivariate Cox regression model (20). According to the median of above risk scores, LUSC patients were divided into high- and low-risk groups. Receiver operating characteristic (ROC) curve was constructed using survivalROC package (version 1.0.3, https://CRAN. $\mathrm{R}$-project.org/package $=$ survivalROC) based on $\mathrm{R}$, and was used to measure the risk prediction rate of lncRNAs between high- and low-risk groups.

\section{Statistical analysis}

All statistical analyses were performed using R software. The comparison of gene expression in LUSC tissues and normal tissues was analyzed using Mean \pm Standard Deviation. T-test was used to estimate the statistical difference, and $\mathrm{P}<0.05$ was considered as significant difference between two groups in expression level.

\section{Results}

\section{Identification of DElncRNA, DEmRNA and DEmiRNA}

After all raw data were normalized, 8,809 lncRNAs, 18,275 mRNAs, and 821 miRNAs were extracted. Using DEGA based on EdgeR package, we identified DElncRNAs, DEmRNAs and DEmiRNAs between LUSC tissues samples and non-LUSC lung tissues samples. According to the $|\log \mathrm{FC}|>2$ and $\mathrm{P}<0.01$ cut-off criteria, a total of 2,114 DElncRNAs (1,667 up- and 447 down-regulated, http:// fp.amegroups.cn/cms/32d9bc8d31415aaab9827d0601d1f bbd/tlcr.2019.09.13-1.pdf), 3,485 DEmRNAs (2,324 upand 1,161 down-regulated, http://fp.amegroups.cn/cms/ a52c08dd78c153449baca4f523871775/tlcr.2019.09.132.pdf), and 175 DEmiRNAs (148 up- and 27 downregulated, http://fp.amegroups.cn/cms/a63f404926063d02 b2c7c606a1972a92/tlcr.2019.09.13-3.pdf) were identified. The distributions of DElncRNAs, DEmRNAs and DEmiRNAs were displayed using volcano plots in Figure $2 A$. The expression levels of DElncRNAs, DEmRNAs and DEmiRNAs between LUSC tissues and non-LUSC normal lung tissues were displayed using heat map in Figure $2 B$.

\section{Co-expression module identification}

All DERNAs including 2,114 DElncRNAs, 3,485 DEmRNAs and 175 DEmiRNAs were used to reconstruct the transcriptional network using WGCNA algorithm. Pearson correlation matrix among genes were converted into a strengthened adjacency matrix by power $\beta=10$ based 

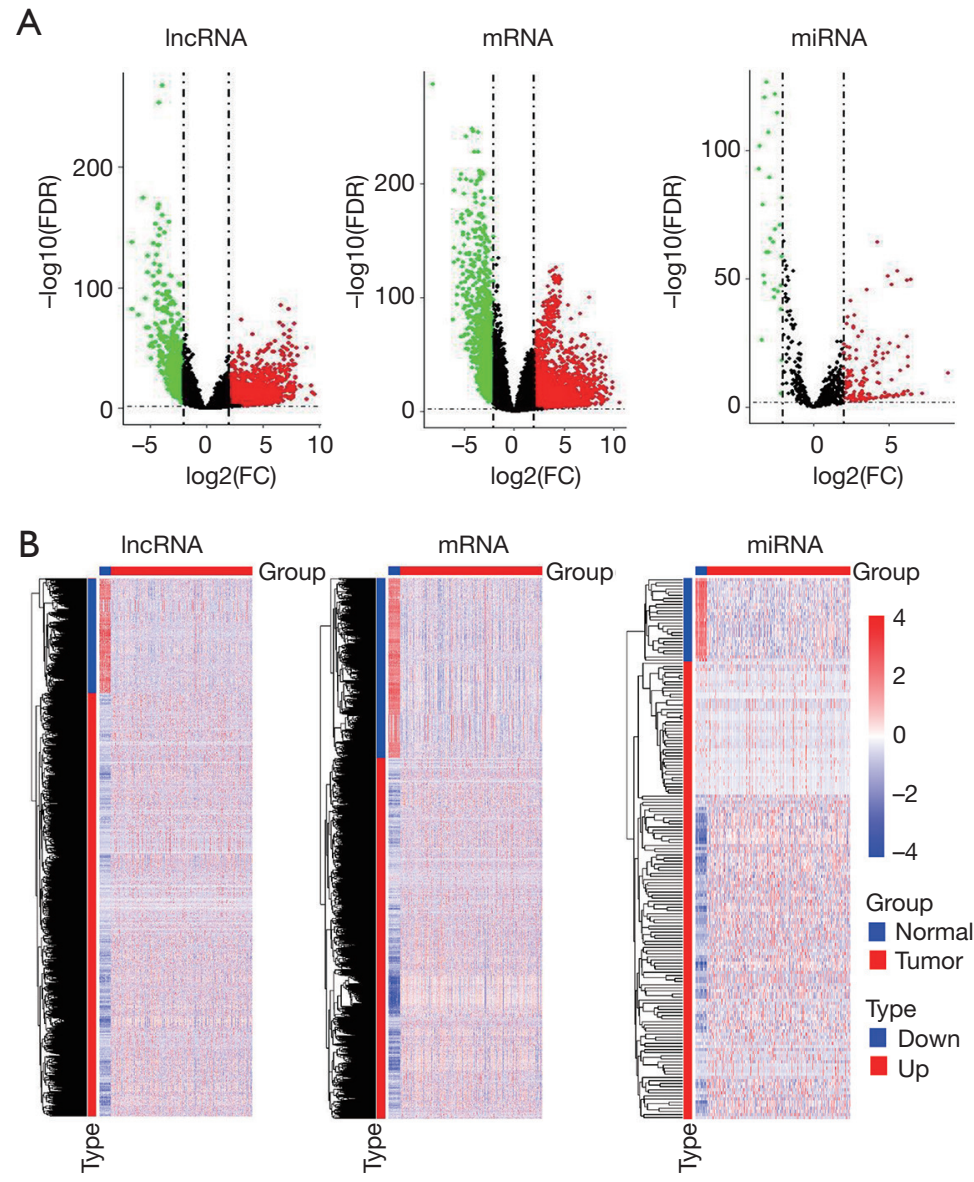

Figure 2 Differentially expressed RNAs in LUSC. (A) Volcano plot of expressions of three types of RNAs. X axis represented the mean expression differences of lncRNAs, mRNAs and miRNAs between LUSC and normal lung tissues samples, and Y axis represented log transformed false discovery rate; (B) heatmap of expressions of three types of differentially expressed RNAs between LUSC and normal lung tissues samples. X-axis represented differentially expressed lncRNAs, mRNAs and miRNAs, and blue represented normal lung tissues samples and red represented LUSC tissues samples. Y-axis represented the samples, and blue represented down-regulated lncRNAs, mRNAs and miRNAs, and red represented up-regulated lncRNAs, mRNAs and miRNAs. The color scale showed the expression values. LUSC, lung squamous cell carcinoma.

on scale-free topology criterion with $\mathrm{R}^{2}=0.94$ (Figure $3 A, B$ ). TOM of each gene pair was calculated, and 16 gene co-expression modules were identified by average linkage hierarchical clustering according to TOM-based dissimilarity measure (1-TOM) (Figure 3C). The correlation analysis between ME and LUSC showed that turquoise module (included 1,694 DElncRNAs and 2,654 DEmRNAs as well as 113 DEmiRNAs, http://fp.amegroups.cn/cms/23ad548482 adf5ccf686ce250556033f/tlcr.2019.09.13-4.pdf) was the most significantly representative module (cor $=-0.9$ and $\mathrm{P}=5 \mathrm{e}-190$ ) (Figure $3 D$ ). The $M M$ in turquoise module possessed the most significant correlation in all modules (cor $=0.99$ and
$\mathrm{P}<1 \mathrm{e}-200$ ) (Figure 3E), and GS analysis across modules showed that turquoise module had the second highest gene significance with the lowest standard deviation (Figure $3 F$ ). Thus, the turquoise module was used for next analyses.

\section{GO and KEGG enrichment analyses}

Based on MM, 660 MEs with $|M M|>0.6$ in turquoise module were selected to investigate the functions by GO and KEGG enrichment analyses. GO enrichment results showed that $216 \mathrm{BPs}, 58 \mathrm{CCs}$ and $33 \mathrm{MFs}$ were significantly enriched. KEGG enrichment analysis showed that 11 

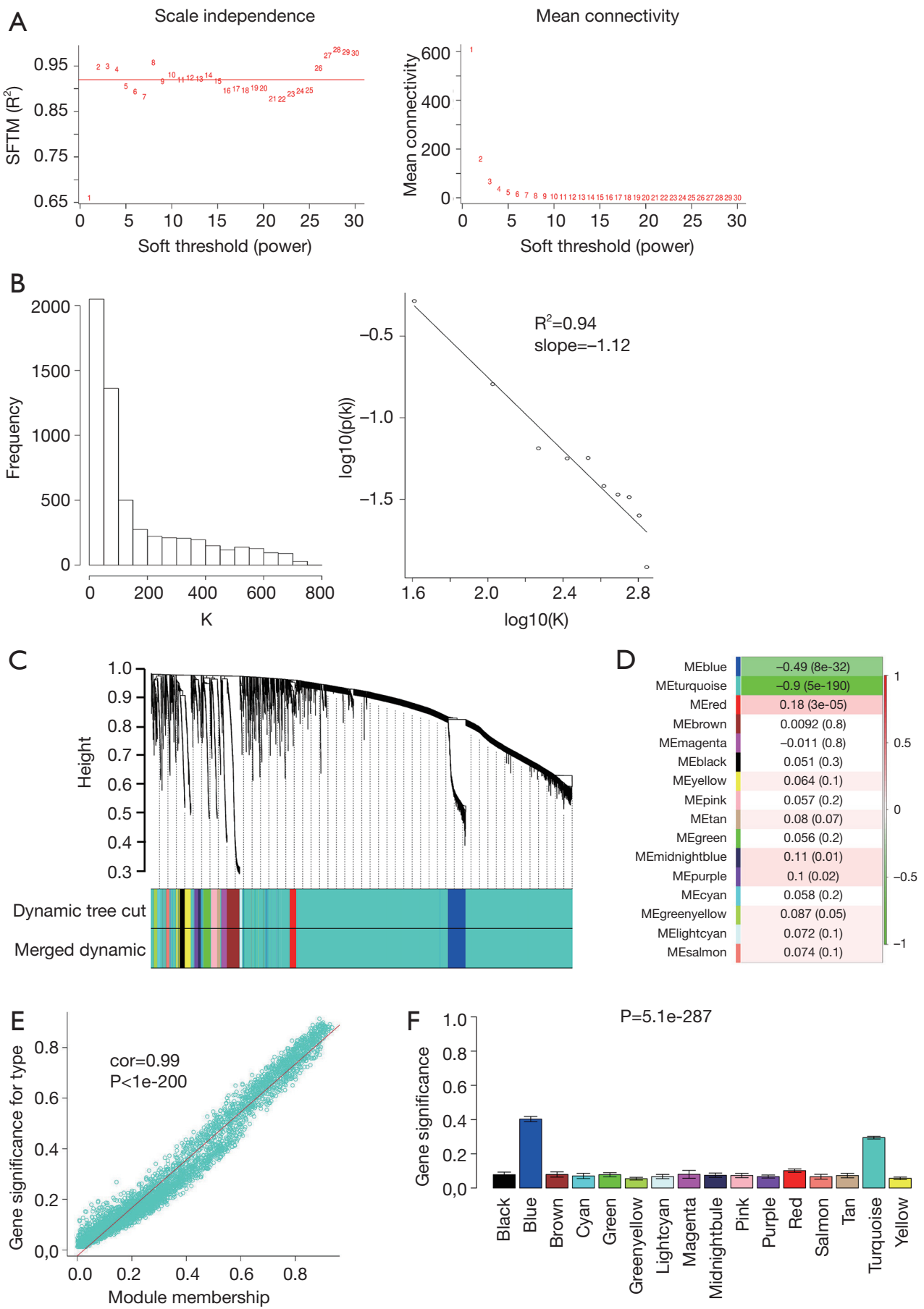

Figure 3 Co-expression network analysis by WGCNA. (A) Analysis of network topology for various soft-threshold powers. (B) The test of property of scale-free network. (C) Identification of LUSC-specific modules. Each short vertical line corresponded to a gene. Each branch represented a expression module of highly interconnected groups of genes. Below the dendrogram, each group of genes was given a color, which indicated its module assignment. Gray suggested that genes were outside all modules. (D) The associations of modules and LUSC. Turquoise module was identified to be the most significant association with LUSC. (E) The associations of turquoise module membership and LUSC. (F) Barplot of mean significance across modules. The higher mean gene significance represented the higher correlation between module and LUSC. LUSC, lung squamous cell carcinoma; WGCNA, weighted gene co-expression network analysis. 
Table 2 Top 10 pathways enriched by GO and KEGG analyses

\begin{tabular}{|c|c|c|c|}
\hline ID & Description & Adj. $P$ value & Count \\
\hline \multicolumn{4}{|c|}{ Biological process (BP) } \\
\hline GO:0023052 & Signaling & 8.15E-12 & 180 \\
\hline GO:0007155 & Cell adhesion & $4.84 \mathrm{E}-10$ & 64 \\
\hline GO:0022610 & Biological adhesion & $4.84 \mathrm{E}-10$ & 64 \\
\hline GO:0007166 & Cell surface receptor linked signaling pathway & $3.15 \mathrm{E}-08$ & 88 \\
\hline GO:0042221 & Response to chemical stimulus & $3.48 \mathrm{E}-08$ & 96 \\
\hline GO:0006952 & Defense response & 4.57E-08 & 54 \\
\hline GO:0006954 & Inflammatory response & 4.57E-08 & 36 \\
\hline \multicolumn{4}{|c|}{ Cellular component (CC) } \\
\hline GO:0044459 & Plasma membrane part & $3.78 \mathrm{E}-24$ & 159 \\
\hline GO:0005887 & Integral to plasma membrane & $2.36 \mathrm{E}-22$ & 112 \\
\hline GO:0031226 & Intrinsic to plasma membrane & $2.36 \mathrm{E}-22$ & 113 \\
\hline GO:0005886 & Plasma membrane & $1.03 E-21$ & 229 \\
\hline GO:0016020 & Membrane & $6.95 \mathrm{E}-16$ & 342 \\
\hline GO:0044421 & Extracellular region part & $1.00 \mathrm{E}-12$ & 82 \\
\hline GO:0044425 & Membrane part & $3.79 \mathrm{E}-12$ & 290 \\
\hline GO:0030246 & Carbohydrate binding & $2.46 \mathrm{E}-08$ & 40 \\
\hline GO:0060089 & Molecular transducer activity & $4.55 \mathrm{E}-07$ & 120 \\
\hline GO:0004871 & Signal transducer activity & $4.55 \mathrm{E}-07$ & 120 \\
\hline GO:0004872 & Receptor activity & 8.65E-07 & 100 \\
\hline GO:0005529 & Sugar binding & $2.39 \mathrm{E}-05$ & 23 \\
\hline GO:0030247 & Polysaccharide binding & $2.50 \mathrm{E}-04$ & 19 \\
\hline GO:0001871 & Pattern binding & $2.50 \mathrm{E}-04$ & 19 \\
\hline GO:0005539 & Glycosaminoglycan binding & $9.16 \mathrm{E}-04$ & 17 \\
\hline GO:0019199 & Transmembrane receptor protein kinase activity & 1.17E-03 & 12 \\
\hline GO:0004888 & Transmembrane receptor activity & 1.17E-03 & 70 \\
\hline
\end{tabular}

Table 2 (continued) 
Table 2 (continued)

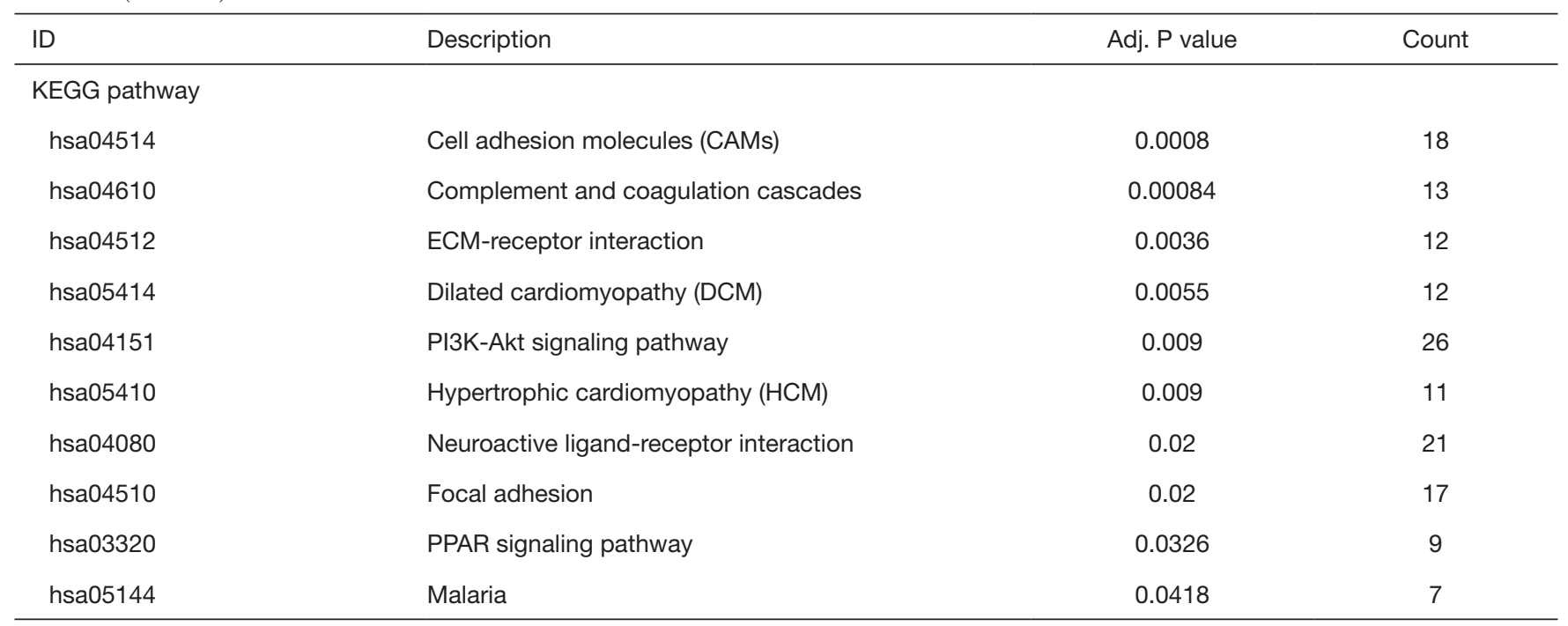

GO, gene ontology; KEGG, Kyoto Encyclopedia of Genes and Genomes.

KEGG pathways were significantly enriched. Top 10 GO terms and KEGG pathways were showed in Table 2, and the most significant BP, CC, MF and KEGG pathway were Signaling (GO:0023052, $\mathrm{P}=8.15 \mathrm{E}-12$ ), Plasma membrane part (GO:0044459, $\mathrm{P}=3.78 \mathrm{E}-24)$, Carbohydrate binding (GO:0030246, P=2.46E-08) and Cell adhesion molecules (hsa04514, $\mathrm{P}=0.0008$ ), separately. In order to elucidate the potentially biological complexities, pathway-gene network was constructed to show the relationships between pathways and genes (Figure 4A). PI3K-Akt signaling pathway contained the most significant genes in the network, which involved in many cellular functions such as transcription, translation, proliferation, growth, and survival.

\section{PPI network construction and key gene validation}

DEmRNAs (MEs) in the most significant turquoise module were used to perform PPI network analysis. Based on MM, 660 MEs with $|M M|>0.6$ (653 positive correlation and 7 negative correlation) were selected to construct PPI network. At minimum required interaction score $=$ the highest confidence 0.9 , a total of 239 among 660 MEs was filtered into PPI network, and a PPI network with 239 nodes and 625 edges was established (Figure 4B). Highly correlated module analysis showed that 3 significantly correlated modules were identified in whole PPI network, and the most significant module (subnetwork 1) included 46 nodes (all genes down-regulated) and 326 edges (Score=14.489) (Figure 4C). Centrality analysis of genes in the subnetwork1 showed that top 3 genes among genes obtained by each Centrality method were FPR2 $(\log \mathrm{FC}=-3.511, \mathrm{P}=1.08 \mathrm{e}-74), G N G 11(\log \mathrm{FC}=-2.994$, $\mathrm{P}=4.22 \mathrm{e}-110)$ and $A D C Y 4(\log \mathrm{FC}=-2.472, \mathrm{P}=1.15 \mathrm{e}-99)$ (Table 3), and the 3 genes were identified as essential genes in subnetwork1. The expression analysis based on GEPIA database showed that the 3 genes were significantly downregulated expressed in LUSC tissues (Figure 4D), and which were consistent with the results obtained in this study. The expression analysis between major pathological stagings showed that FPR2 ( $\mathrm{F}=2.76$ and $\mathrm{P}=0.0419)$ had significant difference between four stages, with higher expression in stage IV (Figure 4E). Survival analysis revealed that low mRNA expression of FPR2 and GNG11 resulted in a higher OS rate than high mRNA expression $(\mathrm{P}=0.036$ and 0.049 , respectively) (Figure $4 F$ ). Pearson correlation analysis showed that mRNA expression between FPR2 and GNG11 $(\mathrm{R}=0.44$ and $\mathrm{P}=0)$ had more similar pattern in LUSC and normal lung tissues (Figure $4 G$ ), which demonstrated that FPR2 and GNG11 possessed the stronger co-expression in LUSC and normal lung tissues. The findings indicate that FPR2 and GNG11 may sever as potential prognosticators of OS in LUSC patients.

\section{Construction of ceRNA network}

The relationships among DElncRNA, DEmiRNA and 


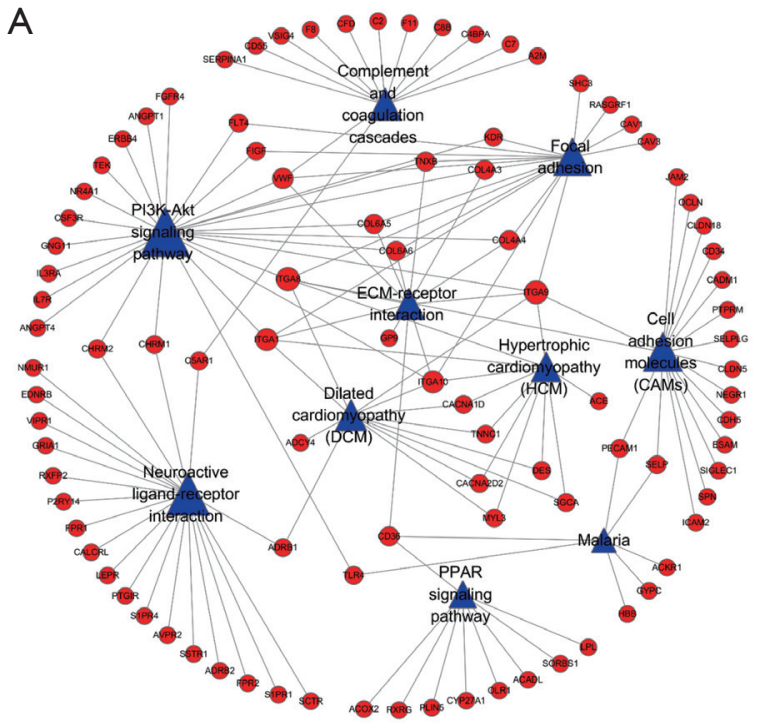

C
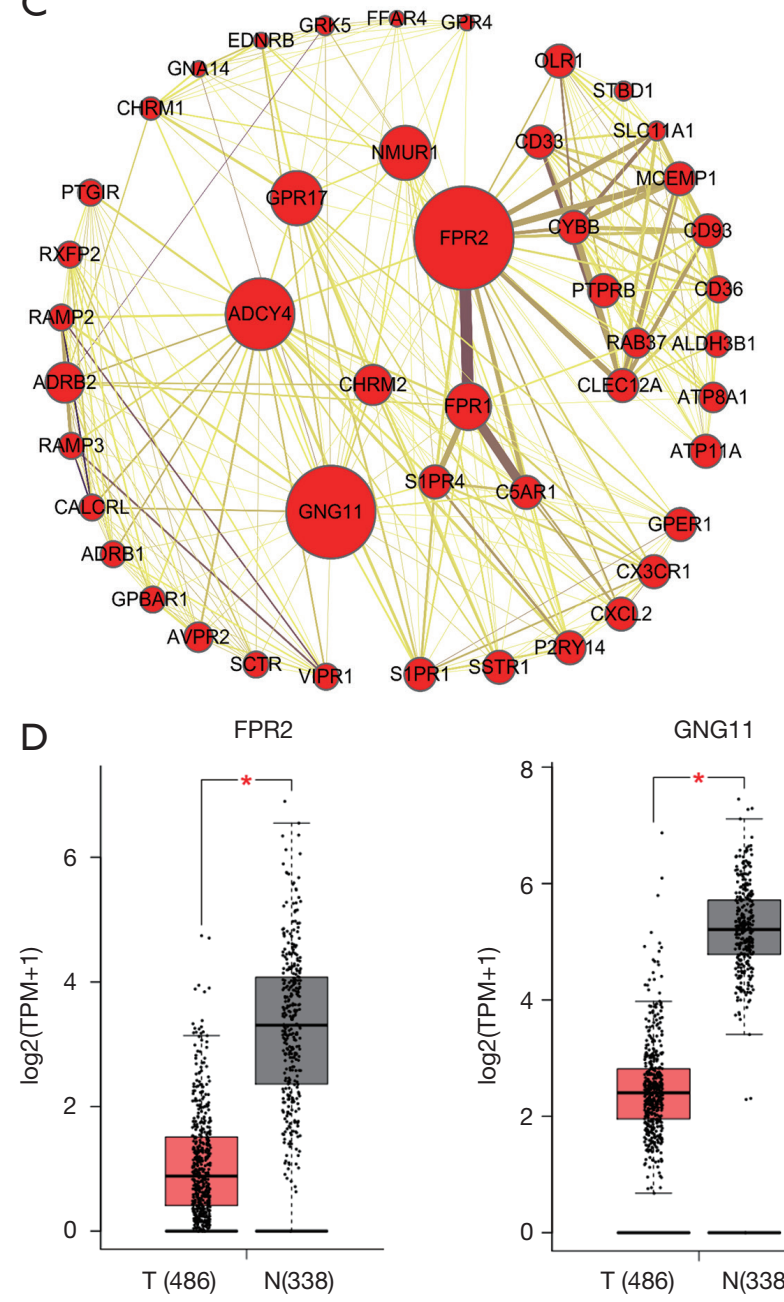

B

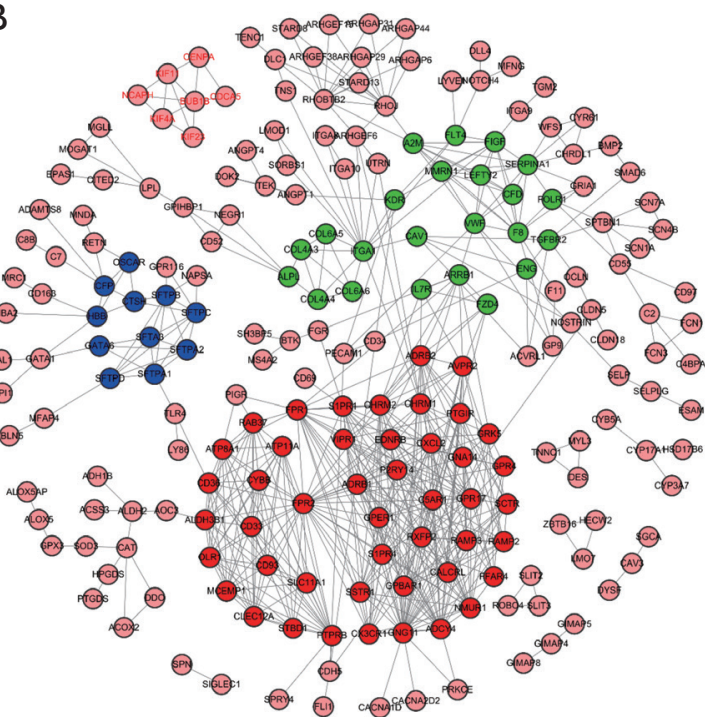

Node

Increasing degree

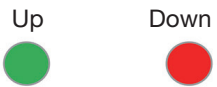

Edge

Increasing

co-expression

Combined score

$0.800 \quad 0.900 \quad 1.000$ 


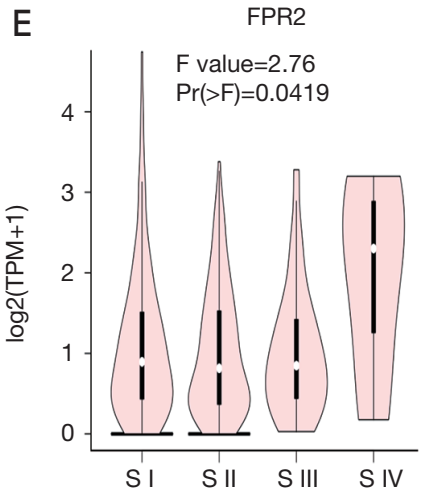

F
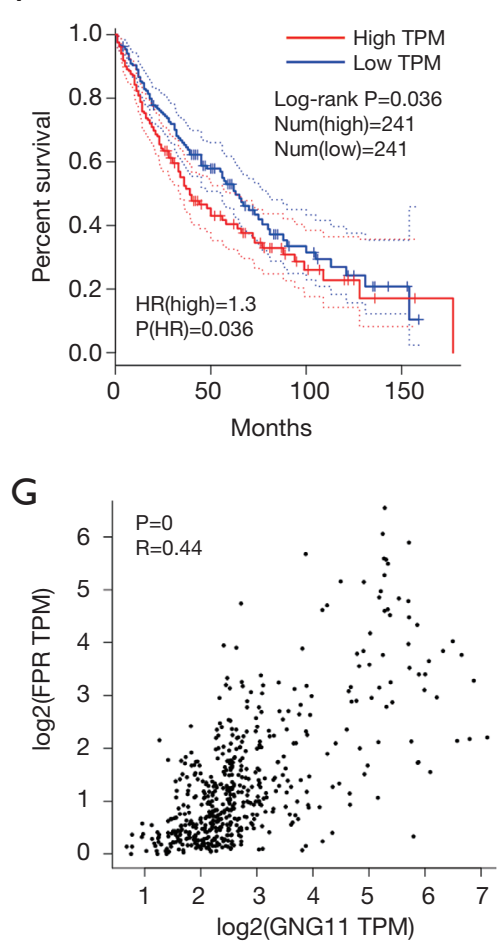

GNG11

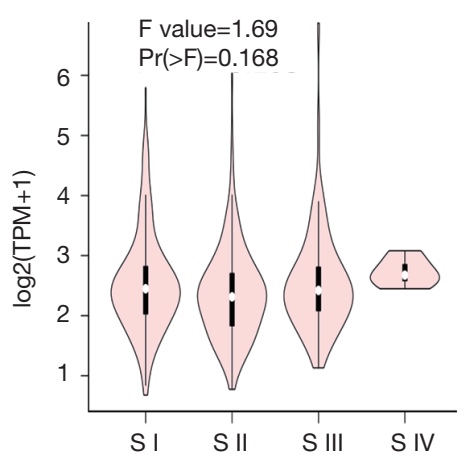

GNG11

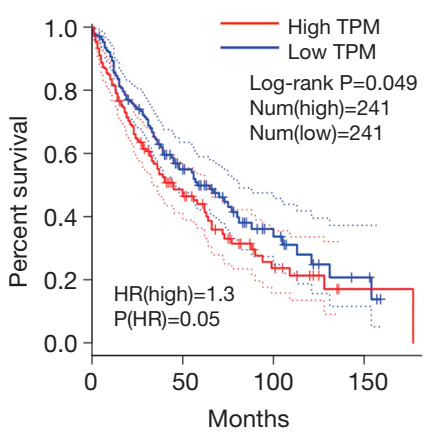

ADCY4

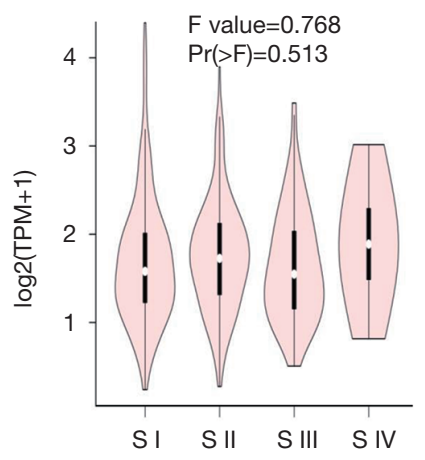

ADCY4

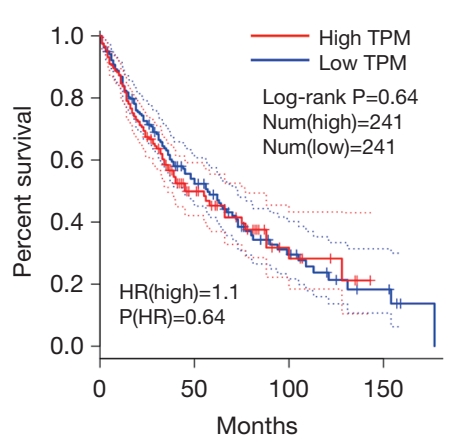

Figure 4 Molecular network and key gene analysis in LUSC. (A) Ten pathways and 97 genes were used to construct pathway-gene network. Red cycle represented gene, and blue triangle represented pathway. (B) PPI network. 239 genes including 232 down-regulated and 7 upregulated genes were used to construct the PPI network. Nodes with red and black labels represented up-regulated and down-regulated genes, respectively. Red, green and blue nodes represented the genes in highly correlated modules by MCODE methods. (C) highly correlated module analysis showed that three subnetworks were identified, and the most significant subnetwork included 46 nodes and 326 edges. Each node represented one gene, and bigger nodes represented genes with more links. Each edge represented the interactive relationship between two genes. Thicker edges represented higher co-expression score among genes and deeper color edges (green to yellow to black) represented higher combined score among genes. (D) The expression of FPR2, GNG11 and ADCY4 mRNAs were significantly down-regulated in LUSC tissues. (E) The expression of FPR2 had significant difference between major pathological stagings, with higher expression in stage IV. (F) Low mRNA expression of FPR2 and GNG11 was significantly associated with a higher overall survival rate of LUSC patients. (G) The mRNA expression between FPR2 and GNG11 had more similar pattern in LUSC and normal tissues. LUSC, lung squamous cell carcinoma; PPI, protein and protein interaction; MCODE, molecular complexity detection; TPM, transcripts per million; HR, hazard ratio. 
Table 3 Top 3 genes with higher scores identified by centrality analyses

\begin{tabular}{llccccccc}
\hline Rank & Gene & Subgragh & Degree & Eigenvector & Information & Betweenness & Closeness & Network \\
\hline 1 & FPR2 & $810,463.75$ & 34 & 0.2978219 & 10.2128935 & 822.15875 & 822.15875 & 32.771732 \\
2 & GNG11 & $771,008.3$ & 31 & 0.29110122 & 9.952475 & 390.3095 & 390.3095 & 30.515686 \\
3 & ADCY4 & $594,200.06$ & 25 & 0.255286 & 9.313109 & 257.04285 & 257.04285 & 23.600285 \\
\hline
\end{tabular}

DEmRNAs were required before lncRNA-miRNAsmRNAs ceRNA network was constructed. Using online miRcode tool, the relationships among 1,694 DElncRNAs and 113 DEmiRNAs in the turquoise module were evaluated, and finally 121 LUSC-specific DElncRNAs (92 up- and 29 down-regulated, Table 4) and 18 LUSCspecific DEmiRNAs (12 up- and 6 down-regulated, Table 4) were putatively identified to interact. Using online tools including TargetScan, miRDB and miRTarBase, we predicted the relationships among DEmiRNAs and their target DEmRNAs. Finally, 3 LUSC-specific target DEmRNAs (1 up- and 2 down-regulated, Table 4) were putatively identified to interact with 18 LUSC-specific DEmiRNAs.

On the basis of the above data, lncRNA-miRNAmRNA ceRNA network was constructed and visualized using Cytoscape software. Based on ceRNA hypothesis and the expression levels of DElncRNA, DEmiRNAs and DEmRNAs, two lncRNA-miRNA-mRNA ceRNA networks including over-expressed (Figure $5 A$ ) and under-expressed (Figure $5 B$ ) networks were constructed. In over-expressed ceRNA network, 65 up-regulated DElncRNAs, 6 downregulated DEmiRNAs and 1 up-regulated DEmRNAs were included and 111 edges were contained. In under-expressed ceRNA network, 23 down-regulated DElncRNAs, 12 upregulated DEmiRNAs and 1 down-regulated DEmRNAs were included and 78 edges were contained.

\section{OS analysis of LUSC-specific lncRNA signature}

The relationships of OS in LUSC patients and DElncRNAs in ceRNA network were determined using KM estimate and LR test. According to $\mathrm{P}<0.05$ cut-off threshold, 8 DElncRNAs including AL391152.1 [P=3.834e-03, HR(high) $=0.66346,95 \%$ CI: 0.4967-0.8763], CACNA2D3-AS1 $[\mathrm{P}=8.581 \mathrm{e}-03, \mathrm{HR}$ (high) $=0.68679,95 \% \mathrm{CI}: 0.5149-$ 0.9095], KCNQ5-IT1 [P=0.013, HR(high) $=0.70386,95 \%$ CI: 0.5266-0.9287], LNX1-AS1 [P=0.03775, HR(high) $=1.34097,95 \%$ CI: 1.016-1.79], MAGI2-AS3 [P=0.01855, $\mathrm{HR}($ high $)=1.39845,95 \%$ CI: 1.0569-1.8601], POU6F2-AS2
[P=9.601e-03, HR(high) $=0.69481,95 \%$ CI: 0.5189-0.9147], SOX2-OT $[\mathrm{P}=0.03924$, HR(high) $=0.74685,95 \%$ CI: $0.5612-$ $0.9865]$ and TTTY16 [P=0.02715, HR(high) $=1.3863,95 \%$ CI: 1.0483-1.8538] were found to be related to OS of LUSC patients (Figure S1).

\section{Establisbment of lncRNA signature prognostic model}

We used univariate regression analysis to identify DElncRNAs associated with the OS of LUSC patients. With $\mathrm{P}<0.05$ cut-off threshold, a group of 10 DElncRNAs including TTTY16 $[\mathrm{P}=0.00583$, HR(high) $=1.155836$, 95\% CI: 1.04276-1.28118], ADAMTS9-AS2 [P=0.01317, $\mathrm{HR}($ high $)=1.15863$, 95\% CI: 1.03132-1.30165], AC006238.1 [P=0.014560, HR(high) $=1.16733,95 \% \mathrm{CI}$ : 1.03107-1.32160], AC123595.1 [P=0.01636, HR(high) $=1.16110,95 \%$ CI: 1.02780-1.31168], CACNA2D3-AS1 $[\mathrm{P}=0.01905, \mathrm{HR}($ high $)=0.87871,95 \% \mathrm{CI}: 0.78878-$ 0.97903], KCNQ5-IT1 [P=0.02881, HR(high) $=0.89105$, 95\% CI: 0.80350-0.98814], POU6F2-AS2 [P=0.03166, $\mathrm{HR}$ (high) $=0.92865$, 95\% CI: 0.86801-0.99352], AL391152.1 [P=0.03411, HR(high) $=0.90647,95 \% \mathrm{CI}$ : 0.82776-0.99267], CHODL-AS1 [P=0.03806, HR(high) $=0.87980,95 \% \mathrm{CI}: 0.77952-0.99297]$ and LINC00462 $[\mathrm{P}=0.04096, \mathrm{HR}($ high $)=0.93351,95 \% \mathrm{CI}: 0.87391-$ 0.99718 ] was detected to have significantly prognostic value (http://fp.amegroups.cn/cms/23ad548482adf5ccf 686ce250556033f/tlcr.2019.09.13-4.pdf). Furthermore, we found that 5 DElncRNAs (TTTY16, CACNA2D3AS1, KCNQ5-IT1, POU6F2-AS2 and AL391152.1) were simultaneously identified to be related to OS in KM (LR test) and univariate regression analysis. The 5 DElncRNAs were fitted into the multivariate Cox regression model with Stepwise method. The result showed that a group of 3 DElncRNAs including TTTY16, POU6F2-AS2 and $C A C N A 2 D 3-A S 1$ had significantly prognostic value with OS of LUSC patients, and 3-lncRNA prognostic model was established $(\mathrm{P}=0.002891)$. High lncRNA expression of POU6F2-AS2 and CACNA2D3-AS1, and low mRNA expression of TTTY16 resulted in a higher OS in 
Table 4 DERNAs in ceRNA network of LUSC

\begin{tabular}{|c|c|c|c|}
\hline RNA & $\log F C$ & $P$ value & FDR \\
\hline \multicolumn{4}{|l|}{ LncRNA } \\
\hline LINC00392 & 9.440110812 & $1.54 \mathrm{E}-14$ & $9.88 \mathrm{E}-14$ \\
\hline LINC00355 & 7.791573469 & $5.43 \mathrm{E}-23$ & $6.90 \mathrm{E}-22$ \\
\hline POU6F2-AS2 & 7.639818861 & $3.74 \mathrm{E}-54$ & $2.29 E-52$ \\
\hline AL513123.1 & 7.295631459 & $9.06 \mathrm{E}-39$ & $2.90 \mathrm{E}-37$ \\
\hline HOTAIR & 6.277782238 & $1.22 \mathrm{E}-17$ & $1.04 \mathrm{E}-16$ \\
\hline MIR137HG & 6.227772241 & $4.95 \mathrm{E}-13$ & 2.77E-12 \\
\hline MIR205HG & 6.149099342 & $1.46 \mathrm{E}-58$ & $1.13 E-56$ \\
\hline LINC00536 & 5.751355879 & $3.13 \mathrm{E}-14$ & $1.96 \mathrm{E}-13$ \\
\hline TTTY20 & 5.58577463 & $2.12 \mathrm{E}-04$ & 4.15E-04 \\
\hline TDRG1 & 5.5076554 & 1.34E-09 & 5.30E-09 \\
\hline SOX2-OT & 5.475037306 & $7.74 \mathrm{E}-21$ & 8.49E-20 \\
\hline LINC00462 & 5.374797677 & $2.26 \mathrm{E}-15$ & $1.56 \mathrm{E}-14$ \\
\hline AC006305.1 & 5.118874308 & $2.10 \mathrm{E}-34$ & 5.34E-33 \\
\hline LSAMP-AS1 & 4.998808 & $9.45 \mathrm{E}-25$ & 1.35E-23 \\
\hline LINC00461 & 4.897041384 & $8.40 \mathrm{E}-14$ & $5.04 \mathrm{E}-13$ \\
\hline PEX5L-AS1 & 4.885905232 & 4.68E-04 & 8.76E-04 \\
\hline LINC00518 & 4.818106321 & $6.29 \mathrm{E}-11$ & $2.89 \mathrm{E}-10$ \\
\hline UCA1 & 4.789191312 & $1.54 \mathrm{E}-15$ & $1.08 \mathrm{E}-14$ \\
\hline IL20RB-AS1 & 4.727889802 & $2.19 \mathrm{E}-16$ & $1.66 \mathrm{E}-15$ \\
\hline PART1 & 4.479053831 & $6.10 \mathrm{E}-17$ & 4.87E-16 \\
\hline LINC00470 & 4.443384297 & $4.63 \mathrm{E}-16$ & $3.38 \mathrm{E}-15$ \\
\hline AL163952.1 & 4.38576249 & 4.12E-14 & $2.55 E-13$ \\
\hline LINC00051 & 4.333975408 & 1.10E-08 & 3.89E-08 \\
\hline KCNQ5-IT1 & 4.246609506 & $1.27 \mathrm{E}-17$ & $1.08 \mathrm{E}-16$ \\
\hline LINC00404 & 4.232371376 & 6.00E-05 & $1.28 \mathrm{E}-04$ \\
\hline SOX21-AS1 & 4.146402175 & $2.14 \mathrm{E}-28$ & $3.84 \mathrm{E}-27$ \\
\hline LINC00308 & 4.053357554 & $2.86 \mathrm{E}-07$ & $8.48 \mathrm{E}-07$ \\
\hline LINC00488 & 3.978445763 & $2.48 \mathrm{E}-05$ & 5.56E-05 \\
\hline AP002478.1 & 3.936160123 & $2.90 \mathrm{E}-15$ & $1.97 \mathrm{E}-14$ \\
\hline AC080129.1 & 3.926928079 & $4.21 \mathrm{E}-08$ & $1.38 \mathrm{E}-07$ \\
\hline GRM5-AS1 & 3.73674902 & $1.10 \mathrm{E}-10$ & $4.88 \mathrm{E}-10$ \\
\hline GDNF-AS1 & 3.643490224 & $4.20 \mathrm{E}-13$ & 2.37E-12 \\
\hline LINC00320 & 3.586351022 & 7.76E-05 & 1.62E-04 \\
\hline AL391152.1 & 3.583026125 & 3.77E-31 & 7.96E-30 \\
\hline LNX1-AS1 & 3.545403022 & 5.17E-09 & 1.91E-08 \\
\hline
\end{tabular}

Table 4 (continued)
Table 4 (continued)

\begin{tabular}{|c|c|c|c|}
\hline RNA & $\log \mathrm{FC}$ & $P$ value & FDR \\
\hline MLIP-IT1 & 3.483310114 & $6.08 \mathrm{E}-17$ & 4.86E-16 \\
\hline LINC00391 & 3.464052328 & $2.83 E-11$ & $1.35 \mathrm{E}-10$ \\
\hline C10orf91 & 3.413159709 & $3.59 E-24$ & 4.94E-23 \\
\hline AC109462.1 & 3.374590902 & $5.81 \mathrm{E}-12$ & $2.96 \mathrm{E}-11$ \\
\hline KIRREL3-AS3 & 3.331565793 & $1.76 \mathrm{E}-05$ & 4.05E-05 \\
\hline CHODL-AS1 & 3.276032463 & 3.33E-10 & $1.41 \mathrm{E}-09$ \\
\hline ADIPOQ-AS1 & 3.160141054 & 2.96E-03 & 4.92E-03 \\
\hline AC016912.1 & 3.08743503 & 7.95E-04 & $1.44 \mathrm{E}-03$ \\
\hline LINC00520 & 3.079503876 & $7.79 \mathrm{E}-11$ & $3.51 \mathrm{E}-10$ \\
\hline C12orf77 & 3.022273593 & 3.30E-05 & 7.30E-05 \\
\hline LINC00052 & 2.997128874 & $2.65 \mathrm{E}-05$ & 5.94E-05 \\
\hline AC100791.1 & 2.994926916 & 7.32E-08 & 2.33E-07 \\
\hline AL139385.1 & 2.99010878 & $3.68 E-28$ & $6.49 \mathrm{E}-27$ \\
\hline DSCAM-AS1 & 2.985792179 & $3.52 E-03$ & 5.80E-03 \\
\hline AC025211.1 & 2.947705948 & $2.26 \mathrm{E}-18$ & $2.06 \mathrm{E}-17$ \\
\hline LINC00460 & 2.945188266 & 5.49E-08 & $1.78 \mathrm{E}-07$ \\
\hline AC012640.1 & 2.940659458 & $2.40 \mathrm{E}-22$ & $2.94 \mathrm{E}-21$ \\
\hline LINC00458 & 2.87315071 & 6.69E-04 & $1.22 \mathrm{E}-03$ \\
\hline LINC00492 & 2.867750469 & $1.36 \mathrm{E}-11$ & $6.65 \mathrm{E}-11$ \\
\hline AC092811.1 & 2.835233349 & 8.16E-09 & 2.93E-08 \\
\hline STEAP2-AS1 & 2.780879429 & $1.79 \mathrm{E}-15$ & $1.25 \mathrm{E}-14$ \\
\hline C11orf44 & 2.766012836 & 4.66E-06 & 1.17E-05 \\
\hline C2orf48 & 2.721907434 & 3.70E-22 & 4.46E-21 \\
\hline LINC00504 & 2.709918399 & $5.66 \mathrm{E}-10$ & 2.33E-09 \\
\hline CACNA2D3-AS1 & 2.685950038 & 3.38E-08 & 1.12E-07 \\
\hline LINC00319 & 2.636763346 & $5.56 \mathrm{E}-08$ & $1.80 \mathrm{E}-07$ \\
\hline LINC00381 & 2.635007306 & 2.17E-08 & 7.39E-08 \\
\hline LINC00184 & 2.623125522 & $1.15 \mathrm{E}-12$ & $6.28 \mathrm{E}-12$ \\
\hline AC006372.1 & 2.616079799 & $1.35 \mathrm{E}-04$ & 2.72E-04 \\
\hline AP000525.1 & 2.59642911 & $2.21 \mathrm{E}-16$ & $1.67 \mathrm{E}-15$ \\
\hline AC018781.1 & 2.586720267 & 1.27E-12 & $6.82 \mathrm{E}-12$ \\
\hline TBL1XR1-AS1 & 2.580626632 & $3.64 \mathrm{E}-14$ & $2.26 \mathrm{E}-13$ \\
\hline AC022762.1 & 2.572878977 & 4.37E-09 & 1.63E-08 \\
\hline SMCR2 & 2.521422296 & $1.07 \mathrm{E}-14$ & $6.93 \mathrm{E}-14$ \\
\hline AC009061.1 & 2.518928136 & 2.67E-09 & $1.02 \mathrm{E}-08$ \\
\hline CLDN10-AS1 & 2.509955326 & 1.44E-03 & $2.50 \mathrm{E}-03$ \\
\hline
\end{tabular}

Table 4 (continued) 
Table 4 (continued)

\begin{tabular}{|c|c|c|c|}
\hline RNA & $\log F C$ & $P$ value & FDR \\
\hline C17orf77 & 2.462406767 & $1.78 \mathrm{E}-03$ & $3.06 \mathrm{E}-03$ \\
\hline AC078802.1 & 2.453881668 & $1.21 \mathrm{E}-17$ & $1.03 \mathrm{E}-16$ \\
\hline AL136131.2 & 2.366865903 & 1.19E-05 & 2.82E-05 \\
\hline WNT5A-AS1 & 2.353778973 & $7.62 \mathrm{E}-14$ & $4.59 \mathrm{E}-13$ \\
\hline PVT1 & 2.349482579 & $2.70 \mathrm{E}-38$ & 8.25E-37 \\
\hline LINC00485 & 2.256897083 & $1.07 \mathrm{E}-05$ & $2.55 \mathrm{E}-05$ \\
\hline AC078778.1 & 2.256314434 & $1.15 \mathrm{E}-28$ & $2.11 \mathrm{E}-27$ \\
\hline IGF2-AS & 2.250318994 & 2.13E-05 & 4.83E-05 \\
\hline RRM1-AS1 & 2.241727965 & $4.80 \mathrm{E}-11$ & $2.24 \mathrm{E}-10$ \\
\hline AC079070.1 & 2.234359786 & $2.23 E-05$ & 5.05E-05 \\
\hline LINC00534 & 2.157428061 & $3.14 \mathrm{E}-05$ & 6.97E-05 \\
\hline AC128709.1 & 2.131149708 & $9.37 \mathrm{E}-07$ & $2.59 \mathrm{E}-06$ \\
\hline AC092422.1 & 2.126433862 & $8.71 \mathrm{E}-06$ & $2.11 \mathrm{E}-05$ \\
\hline LINC00173 & 2.124251844 & $4.73 E-14$ & $2.90 \mathrm{E}-13$ \\
\hline STEAP3-AS1 & 2.11620078 & $5.21 \mathrm{E}-12$ & 2.67E-11 \\
\hline AC097717.1 & 2.10623016 & $1.46 \mathrm{E}-13$ & $8.59 \mathrm{E}-13$ \\
\hline MTUS2-AS1 & 2.099780402 & $2.59 \mathrm{E}-07$ & 7.75E-07 \\
\hline AC002511.1 & 2.065525454 & 5.35E-05 & $1.15 \mathrm{E}-04$ \\
\hline AC073352.1 & 2.049896234 & $1.28 \mathrm{E}-15$ & $9.10 \mathrm{E}-15$ \\
\hline LINC00525 & 2.046446311 & $2.80 \mathrm{E}-08$ & $9.44 \mathrm{E}-08$ \\
\hline C8orf49 & 2.046391517 & $1.02 \mathrm{E}-03$ & $1.82 \mathrm{E}-03$ \\
\hline KCNQ1-AS1 & -2.034335916 & $3.00 \mathrm{E}-18$ & $2.68 \mathrm{E}-17$ \\
\hline AC006238.1 & -2.133822774 & $2.43 E-37$ & 7.07E-36 \\
\hline LINC00469 & -2.175169274 & $7.52 \mathrm{E}-15$ & 4.93E-14 \\
\hline AC123595.1 & -2.179056388 & $1.41 \mathrm{E}-34$ & 3.62E-33 \\
\hline LINC00113 & -2.190134588 & 8.01E-09 & 2.89E-08 \\
\hline TTTY16 & -2.194922826 & 5.23E-09 & $1.93 \mathrm{E}-08$ \\
\hline LINC00472 & -2.232889162 & $3.70 \mathrm{E}-39$ & $1.20 \mathrm{E}-37$ \\
\hline MAGI2-AS3 & -2.290846084 & $6.84 \mathrm{E}-56$ & $4.71 \mathrm{E}-54$ \\
\hline TLR8-AS1 & -2.345990308 & $3.50 \mathrm{E}-14$ & $2.18 \mathrm{E}-13$ \\
\hline AL139819.1 & -2.376701055 & $1.81 \mathrm{E}-20$ & $1.94 \mathrm{E}-19$ \\
\hline BCRP3 & -2.410412265 & $2.43 E-51$ & $1.35 \mathrm{E}-49$ \\
\hline KIAA0087 & -2.416352287 & $2.59 \mathrm{E}-16$ & $1.95 \mathrm{E}-15$ \\
\hline AC093510.2 & -2.423740876 & $8.69 E-42$ & $3.11 \mathrm{E}-40$ \\
\hline MED4-AS1 & -2.437359227 & 2.26E-72 & $2.66 \mathrm{E}-70$ \\
\hline NKX2-1-AS1 & -2.511076899 & $3.48 \mathrm{E}-18$ & $3.10 \mathrm{E}-17$ \\
\hline
\end{tabular}

Table 4 (continued)
Table 4 (continued)

\begin{tabular}{|c|c|c|c|}
\hline RNA & $\log F C$ & $P$ value & FDR \\
\hline LINC00211 & -2.672798113 & 4.11E-27 & $6.76 \mathrm{E}-26$ \\
\hline AC025431.1 & -2.68572414 & $3.16 \mathrm{E}-20$ & 3.30E-19 \\
\hline ATP13A4-AS1 & -2.744528113 & $1.40 \mathrm{E}-14$ & $9.01 \mathrm{E}-14$ \\
\hline C5orf64 & -2.871131256 & $4.04 \mathrm{E}-57$ & 2.94E-55 \\
\hline MY016-AS1 & -3.043363778 & $1.57 \mathrm{E}-31$ & $3.41 \mathrm{E}-30$ \\
\hline AC135178.1 & -3.080541278 & $2.75 \mathrm{E}-45$ & $1.15 \mathrm{E}-43$ \\
\hline AP000438.1 & -3.256244279 & 1.93E-57 & $1.43 E-55$ \\
\hline ADAMTS9-AS2 & -3.624852381 & 2.63E-149 & $2.32 E-146$ \\
\hline LINC00163 & -3.64214957 & $2.30 E-45$ & $9.78 \mathrm{E}-44$ \\
\hline RMST & -3.882619917 & $6.84 \mathrm{E}-41$ & 2.36E-39 \\
\hline ADAMTS9-AS1 & -4.088690083 & $2.87 \mathrm{E}-121$ & 1.33E-118 \\
\hline AC105206.1 & -4.356484234 & $1.54 \mathrm{E}-91$ & 3.31E-89 \\
\hline AL355388.1 & -4.517353966 & $6.41 \mathrm{E}-105$ & $1.82 \mathrm{E}-102$ \\
\hline SFTA1P & -4.619645817 & $6.58 \mathrm{E}-113$ & $2.42 \mathrm{E}-110$ \\
\hline \multicolumn{4}{|l|}{ miRNA } \\
\hline hsa-mir-31 & 5.603226184 & $2.45 E-25$ & $2.61 \mathrm{E}-24$ \\
\hline hsa-mir-205 & 5.575113876 & 2.63E-55 & $9.01 \mathrm{E}-54$ \\
\hline hsa-mir-301b & 4.29013536 & $2.10 \mathrm{E}-28$ & $2.61 \mathrm{E}-27$ \\
\hline hsa-mir-210 & 4.244884194 & $9.62 E-67$ & 4.94E-65 \\
\hline hsa-mir-9 & 4.155804934 & 8.69E-19 & $6.37 \mathrm{E}-18$ \\
\hline hsa-mir-383 & 3.246759952 & 7.18E-05 & $1.48 \mathrm{E}-04$ \\
\hline hsa-mir-183 & 3.218906108 & $3.32 \mathrm{E}-31$ & $4.55 \mathrm{E}-30$ \\
\hline hsa-mir-96 & 2.681966878 & $7.56 \mathrm{E}-33$ & $1.07 \mathrm{E}-31$ \\
\hline hsa-mir-137 & 2.672308656 & 5.19E-06 & 1.27E-05 \\
\hline hsa-mir-25 & 2.204516321 & $1.24 \mathrm{E}-10$ & $4.95 \mathrm{E}-10$ \\
\hline hsa-mir-503 & 2.154171477 & 2.59E-20 & 2.03E-19 \\
\hline hsa-mir-182 & 2.000460902 & $2.85 \mathrm{E}-22$ & $2.54 \mathrm{E}-21$ \\
\hline hsa-mir-140 & -2.055995223 & $5.29 \mathrm{E}-61$ & $2.29 E-59$ \\
\hline hsa-mir-218 & -2.130060686 & $1.14 \mathrm{E}-49$ & $2.83 E-48$ \\
\hline hsa-mir-206 & -2.166983977 & $2.62 \mathrm{E}-06$ & 6.71E-06 \\
\hline hsa-mir-101 & -2.380509751 & $1.16 \mathrm{E}-117$ & $2.38 \mathrm{E}-115$ \\
\hline hsa-mir-338 & -2.631345685 & $6.13 E-67$ & $3.35 E-65$ \\
\hline hsa-mir-144 & -3.09914232 & $9.64 \mathrm{E}-63$ & $4.55 E-61$ \\
\hline \multicolumn{4}{|l|}{ mRNA } \\
\hline PAX3 & 6.151717371 & $1.87 \mathrm{E}-17$ & $7.95 \mathrm{E}-17$ \\
\hline LIMCH1 & -3.424019142 & $1.20 \mathrm{E}-117$ & $1.47 \mathrm{E}-115$ \\
\hline UTRN & -2.012223181 & $4.68 \mathrm{E}-83$ & $2.25 E-81$ \\
\hline
\end{tabular}


A

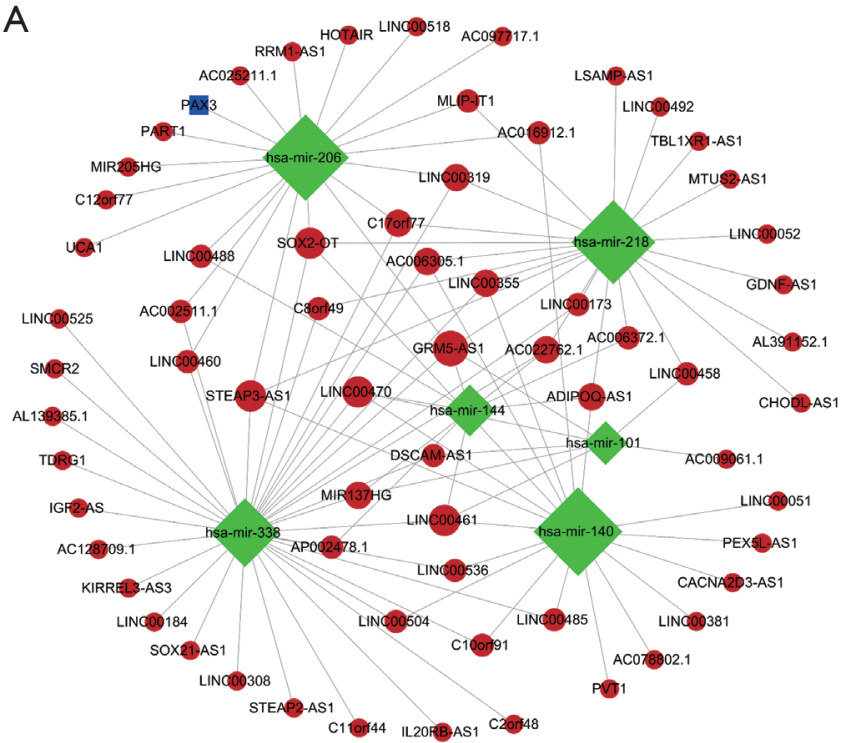

B

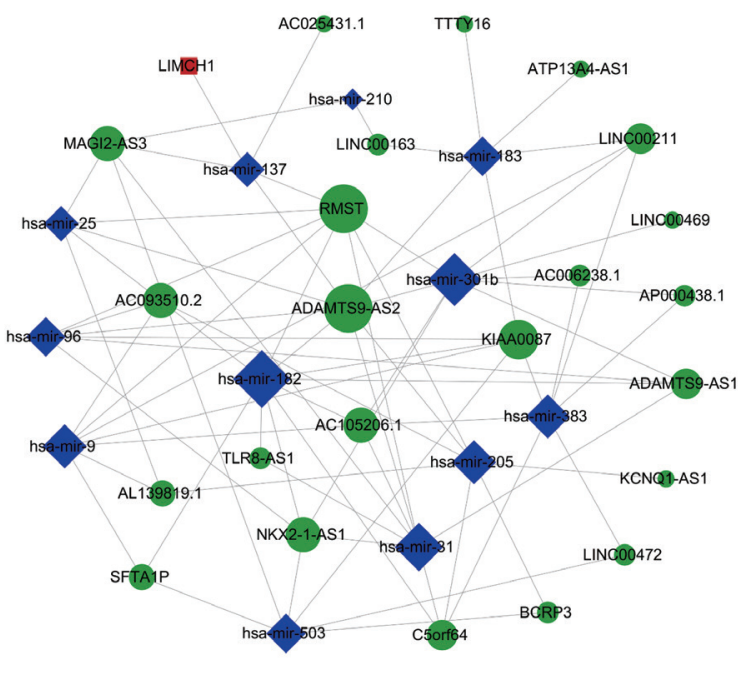

Figure 5 LncRNA-miRNA-mRNA ceRNA network. (A) Over-expressed network. Red circles represented up-regulated lncRNAs, green diamonds represented down-regulated miRNAs, and blue rectangles represented up-regulated mRNAs. (B) Under-expressed network. Green circles represented down-regulated lncRNAs, blue diamonds represented up-regulated miRNAs and red rectangles represented down-regulated mRNAs. ceRNA, competitive endogenous RNA.

patients (Figure 6A). With the risk score of each patient (http://fp.amegroups.cn/cms/d5fa0921ac865b7ce793c7 $183465233 \mathrm{~d} / \mathrm{tlcr} .2019 .09 .13-5 . p d f)$, the LUSC patients were divided into high- and low-risk groups (Figure 6B,C). Comparing to low risk group, the mortality rate of patients in high-risk group was significantly higher $[\mathrm{P}=1 \mathrm{e}-05$, HR(high) $=1.8574,95 \%$ CI: 1.4149-2.5091], and highrisk group was related with worse prognosis (Figure $6 D$ ). The ROC of 3-year survival correlation of the 3-lncRNA signature was analyzed, and the area under the curve (AUC) was computed. The AUC of 3-lncRNA signature was 0.629 (Figure 6E), which indicates its effectiveness as a prognostic biomarker in predicting OS of LUSC patients.

The expression levels of the 3 lncRNAs were analyzed between LUSC tissues and normal lung tissues and between high- and low- risk patient groups. CACNA2D3$A S 1$ and POU6F2-AS2 lncRNAs were significantly highly expressed in LUSC patients $(\mathrm{P}=2.136 \mathrm{e}-12$ and $<2.2 \mathrm{e}$ 16, separately, Figure $7 A)$ with low risk scores $(\mathrm{P}<2.2 \mathrm{e}-16$ and $=1.959 \mathrm{e}-06$, separately, Figure $7 B)$. Whereas, TTTY16 lncRNA was significantly lowly expressed in LUSC patients $(\mathrm{P}=4.592 \mathrm{e}-07$, Figure $7 A)$ with high risk scores $(\mathrm{P}=1.429 \mathrm{e}-14$, Figure $7 B)$. Further, the expressions of three DElncRNAs between dead and alive groups were observed, and showed that the expression patterns of three DElncRNAs between dead and alive groups were separately consistent with that between LUSC and normal tissues and between low and high-risk groups (Figure $7 C$ ). The expression of $C A C N A 2 D-A S 1$ in alive group was significantly higher than that in dead group $(\mathrm{P}=0.002532$, Figure 7C). The expressions of the 3 DElncRNAs in highand risk-groups and survival status groups were showed in Figure 7D.

\section{Discussion}

LUSC is a common malignant cancer, and per year causes hundreds of thousands of deaths. Identifying specific diagnostic and prognostic biomarkers may contribute to improving survival rate among LUSC patients. To improve clinical outcomes, it is essential to identify LUSCrelated prognostic signatures that predict those outcomes. Increasing evidence indicates that lncRNAs play key roles in many biological processes including cancer initiation and progression by ceRNA mechanism. In this study, we systematically analyzed the LUSC-related RNA-seq data from TCGA database by bioinformatics methods including WGCNA, GO and KEGG pathway analyses, PPI network 
A

CACNA2D3-AS1

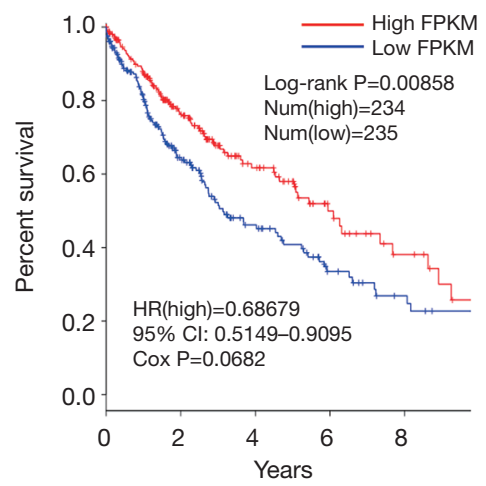

POU6F2-AS2

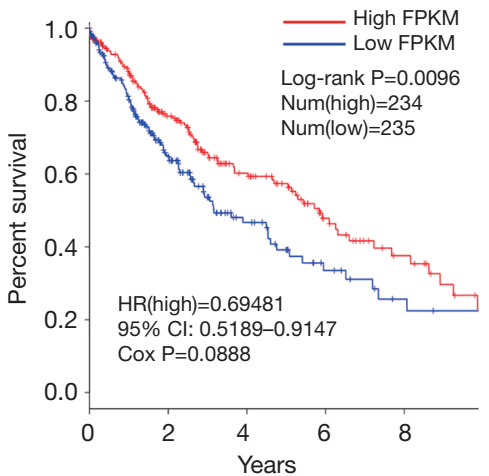

TTTY16
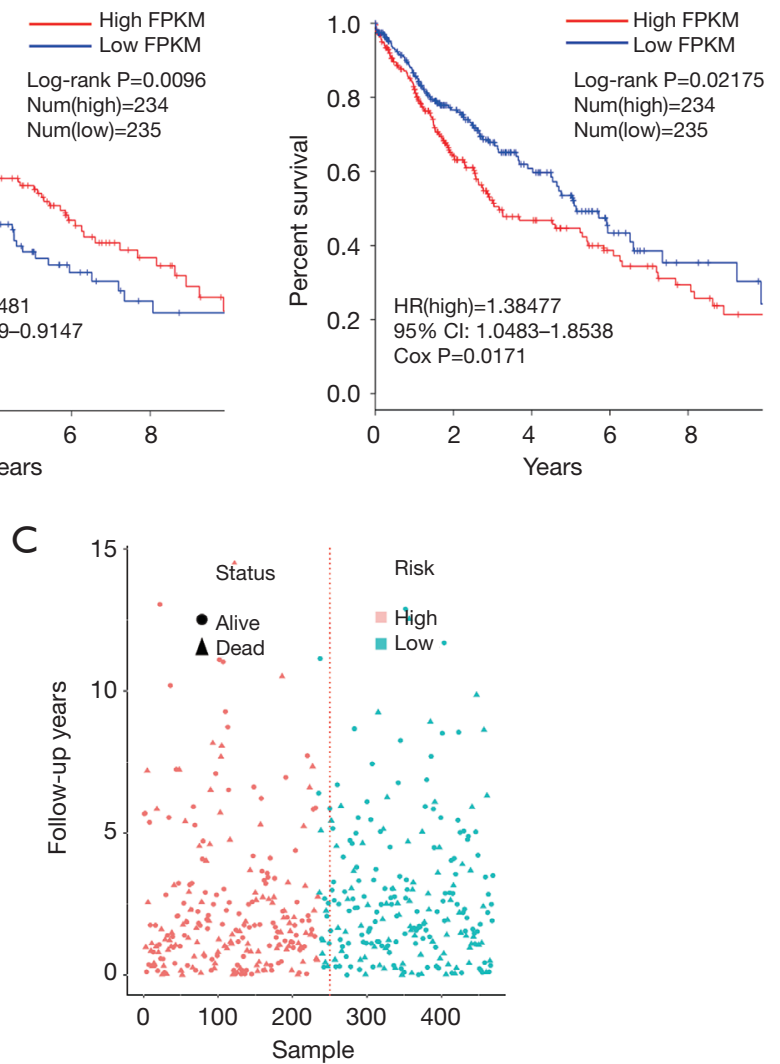

$E$

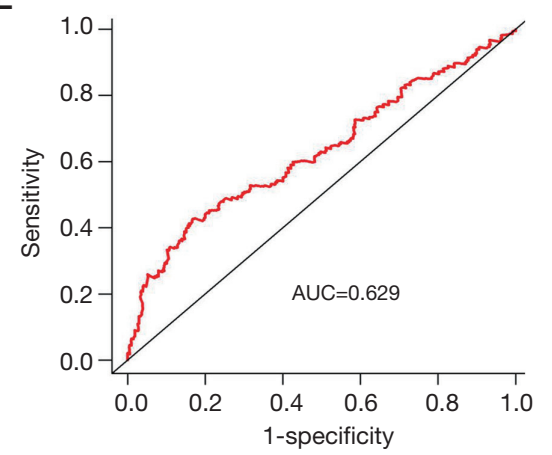

Figure 6 Kaplan-Meier survival curves and the outcome of 3-IncRNA signature. (A) Kaplan-Meier survival cures for 3 lncRNAs associated with overall survival of lung squamous cell carcinoma (LUSC) patients. Log-rank test was used to evaluate the survival differences between low- and high-expression groups. X-axis represented overall survival time (years) and Y-axis represented survival function. (B) The LUSC patients were divided into high- and low-risk groups according to risk scores of LUSC patients. (C) The survival information between highand low-risk groups was plotted using ggplot2 package. (D) Survival analysis showed high risk group had worse prognosis in LUSC patients $(\mathrm{P}=1 \mathrm{e}-05)$. (E) ROC curve showed that AUC of 3-lncRNA prognostic model was 0.629. LUSC, lung squamous cell carcinoma; ROC, receiver operating characteristic; AUC, area under the curve; HR, hazard ratio; $\mathrm{CI}$, confidence interval. 
A

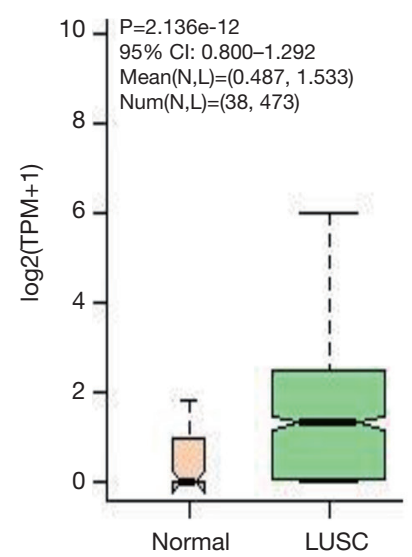

B

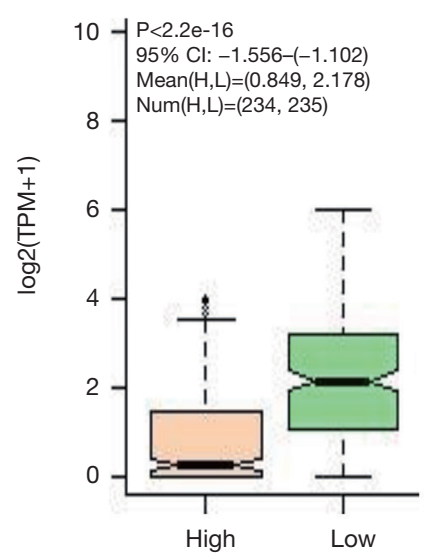

POU6F2-AS2

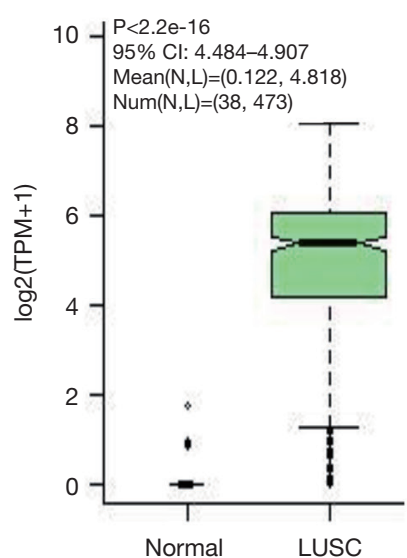

POU6F2-AS2

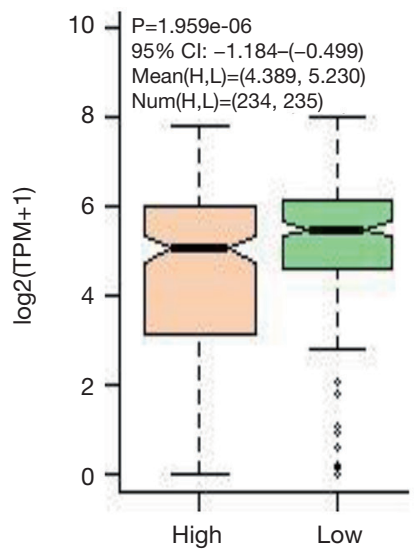

TTTY16

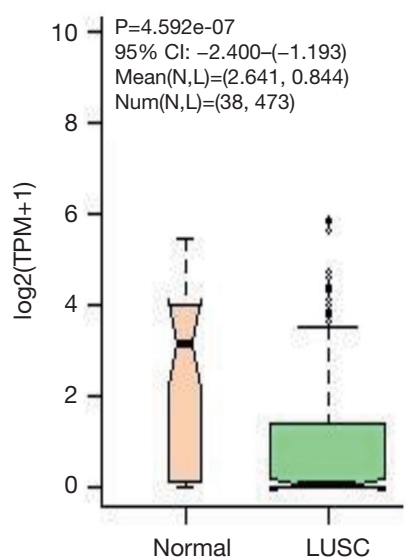

TTTY16

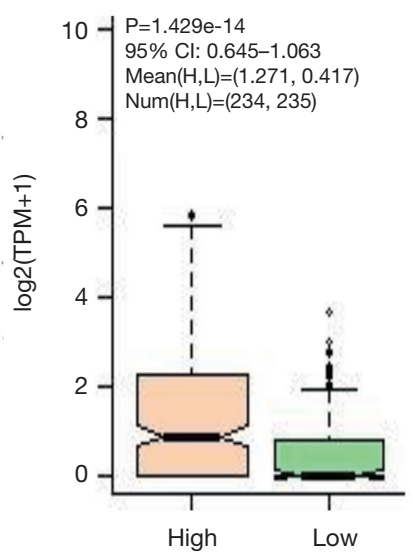

C

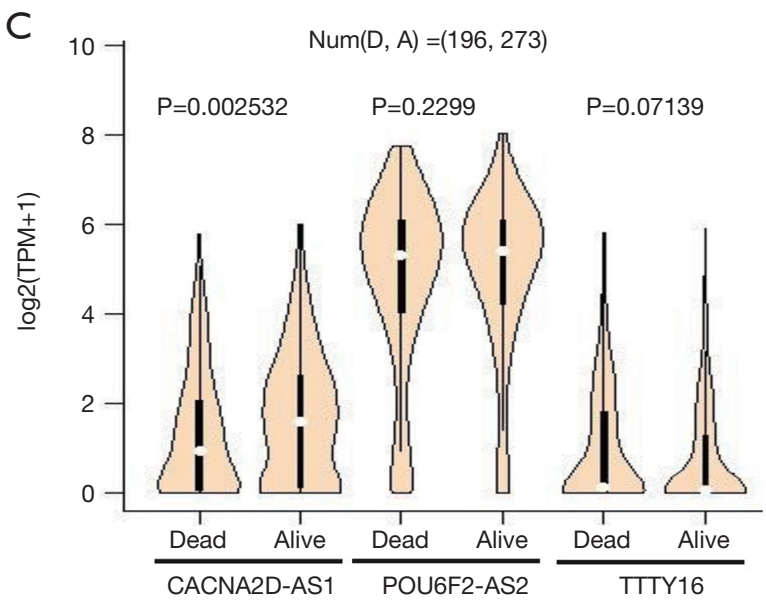

D

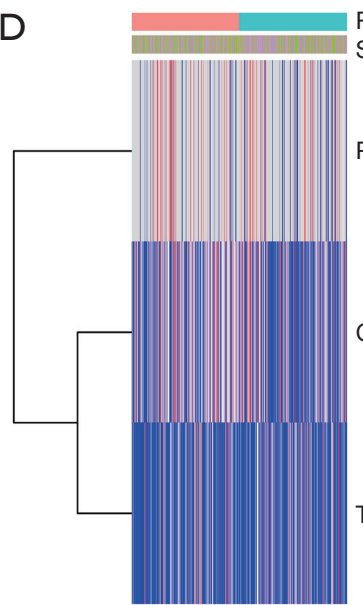

Risk Status POU6F2-AS2<smiles>[10BH]</smiles>

CACNA2D3-AS1

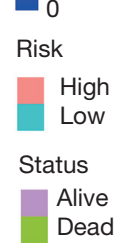

Figure 7 Expression pattern of the 3-lncRNA. (A) The expression pattern of the 3-lncRNA was in LUSC and normal tissues samples; (B) the expression pattern of 3-lncRNA was in high- and low-risk groups; (C) the expression distribution of 3-lncRNA was in alive and dead patients; (D) the expression level of 3-lncRNA was in experimental tissues samples. LUSC, lung squamous cell carcinoma; CI, confidence interval. 
construction, ceRNA network construction and survival analysis, and identified the key genes and key lncRNA signature associated with OS in LUSC patients. Finally, two genes including FPR2 and GNG11 in PPI network, and three lncRNA signature including CACNA2D3$A S 1, P O U 6 F 2-A S 2$ and TTTY16 in ceRNA network were identified to associate with OS in LUSC patients.

FPR2 is an important member of 7 transmembrane G-protein-coupled FPR family, and which was originally identified to play critical roles in host defense (40). Recently, FPR 2 has been reported to play a key role in human diseases such as cancers $(41,42)$. For example, a study found that FPR 2 promoted invasion and metastasis of gastric cancer cells, and negative FPR2 expression was associated with a higher OS of patients (41), which was consistent with our results. GNG11 is a member of guanine nucleotide-binding protein ( $G$ protein) gamma family, and play a role in transmembrane signaling system. Recent studies showed that GNG11 played roles in regulating cell growth and cellular senescence $(43,44)$. So far, the functions of GNG11 is entirely unknown in cancer. No studies have so far reported any association of two genes including FPR2 and GNG11 with LUSC. This study is the first to show aberrant expressions of FPR2 and GNG11 were identified to associate with OS of LUSC patients, and the low mRNA expressions of FPR2 and GNG11 resulted in a higher OS rate in LUSC patients.

In recent years, some studies have investigated ceRNA in LUSC, and constructed ceRNA network to identify some IncRNAs associated with OS of LUSC patients $(9,26,27)$. For example, Sui et al. constructed lcnRNA regulatory ceRNA by integrating analyzing RNA-seq data from TCGA database using DEGA and ceRNA network, and constructed a 22 lncRNAs multivariated Cox regression model and finally identified two lncRNAs including FMO6P and PRR26 had significantly prognostic value (26). In addition, Ning et al. also used LUSC-related from TCGA database to construct lncRNA-miRNA-mRNA ceRNA network, and identified six ceRNAs (PLAU, miR-31-5p, miR-455-3p, FAM83A-AS1, MIR31HG, and MIR99AHG) significantly correlated with survival by survival analysis based on KM method (9). These LUSC-related ceRNA studies provide novel knowledge for a better understanding the ceRNA interactive mechanism in LUSC biology, and contribute to improving the diagnosis and prognosis of LUSC patients. However, few lncRNA-related ceRNA network studies considered the co-expression among ceRNAs. In this study, we firstly used WGCNA to identify the co-expression modules in LUSC, and further constructed lncRNAmiRNA-mRNA ceRNA network based on co-expression genes in the most significant turquoise module according to ceRNA theory. Furthermore, KM (LR test) and univariate Cox regression analysis revealved that 5 DElncRNAs in ceRNA network (TTTY16, CACNA2D3-AS1, KCNQ5IT1, POU6F2-AS2 and AL391152.1) were simultaneously identified to associate with OS of LUSC patients. Multivariate Cox regression analysis showed significant prognostic value of 3 of those lncRNAs (CACNA2D3-AS1, POU6F2-AS2 and TTTY16) in the OS of LUSC patients. A cumulative risk score of the 3-lncRNA showed that 3 -IncRNA signature could independently predict OS in LUSC patients.

Among identified 3-lncRNA signature, POU6F2$A S 2 \operatorname{lncRNA}$ is an antisense lncRNA, and has so far been reported to play role in esophageal squamous cell carcinoma by modulating DNA repair (45). However, no studies reported the association between POU6F2-AS2 and OS of cancer-related patients. In our study, we found that POU6F2-AS2 was highly expressed in LUSC tissue, and LUSC patients with high expression possessed low risk scores. Similar to POU6F2-AS2, CACNA2D3-AS1 is an antisense lncRNA, and no studies so far has reported any association of $C A C N A 2 D 3-A S 1$ with cancer. In our study, we noticed that $C A C N A 2 D 3-A S 1$ in ceRNA network was up-regulated and competed with the down-regulated hasmir-140. Some previous studies showed that hsa-mir-140 often functioned as a tumor suppressor in cancer (46-48). Down-regulated hsa-mir-140 has been suggested to enhance cell proliferation and invasion in colorectal carcinoma and squamous cell LC $(46,47)$, and low expression of hsa-mir-140 is associated with poor prognosis of spinal chordoma (49). This study firstly showed aberrant expression of CACNA2D3-AS1 in LUSC, and indicated that $C A C N A 2 D 3-A S 1$ might sever as a potentially prognostic marker in LUSC. In addition, we observed that TTTY16 in ceRNA network was down-regulated in LUSC, and TTTY16 with low expression competed with upregulated hsa-mir-183. TTTY16 has so far been reported to be associated with cancer (5), and a 14-gene expression signature including TTTY16 was related to the prognoses of LUSC patients (5). Previous studies also showed that hsa-mir-183 was highly expressed in cancer, and promoted tumor cell growth and migration (50,51), which indicated that the high expression of hsa-mir-183 was related to the poor prognosis. So far, no studies about TTTY16 regulating cancer initiation and progression by competing with has- 
mir-183 was reported. In this study, we are the first to report the role of TTTY16 by competing with hsa-mir-183 in LUSC, and low expression of TTTY16 was associated with good prognosis in LUSC. This study findings indicate that CACNA2D3-AS1 and POU6F2-AS2 may play roles as LUSC suppressors, and TTTY16 may function as LUSC promoter.

Despite the findings in clinical implications, some limitations must be noted. Firstly, the findings are obtained based on the TCGA database by pure bioinformatics methods. Although some findings have been validated by GEPIA database, the findings must be verified by experimental methods. Secondly, the 3 prognosticators of 3-lncRNA signature need be evaluated by a median followup duration (3 to 5 years).

\section{Conclusions}

Taken together, three lncRNAs were identified as a potentially prognostic lncRNA signature for LUSC patients. The findings provide novel insights into the lncRNA-related regulatory mechanism by ceRNA network in LUSC. Further, more functional studies are required to elucidate the lncRNA-related molecular mechanisms underlying LUSC.

\section{Acknowledgments}

Funding: This work was supported by National Natural Science Foundation of China (no 31660655), Yunnan Province Applied Basic Research Projects (nos 2016FB146, 2017FB042, 2018FE001) and Fund from Health and Family Planning Commission of Yunnan Province (no 2017NS261).

\section{Footnote}

Conflicts of Interest: The authors have no conflicts of interest to declare.

Ethical Statement: The authors are accountable for all aspects of the work in ensuring that questions related to the accuracy or integrity of any part of the work are appropriately investigated and resolved.

\section{References}

1. Bray F, Ferlay J, Soerjomataram I, et al. Global cancer statistics 2018: GLOBOCAN estimates of incidence and mortality worldwide for 36 cancers in 185 countries. CA Cancer J Clin 2018;68:394-424.

2. Blandin Knight S, Crosbie PA, Balata H, et al. Progress and prospects of early detection in lung cancer. Open Biol 2017;7. doi: 10.1098/rsob.170070.

3. Ling DJ, Chen ZS, Liao QD, et al. Differential effects of MTSS1 on invasion and proliferation in subtypes of non-small cell lung cancer cells. Exp Ther Med 2016;12:1225-31.

4. Gandara DR, Hammerman PS, Sos ML, et al. Squamous cell lung cancer: from tumor genomics to cancer therapeutics. Clin Cancer Res 2015;21:2236-43.

5. Li J, Wang J, Chen Y, et al. A prognostic 4-gene expression signature for squamous cell lung carcinoma. J Cell Physiol 2017;232:3702-13.

6. Tanoue LT, Detterbeck FC. New TNM classification for non-small-cell lung cancer. Expert Rev Anticancer Ther 2009;9:413-23.

7. Nesbitt JC, Putnam JB, Jr., Walsh GL, et al. Survival in early-stage non-small cell lung cancer. Ann Thorac Surg 1995;60:466-72.

8. Campbell JD, Alexandrov A, Kim J, et al. Distinct patterns of somatic genome alterations in lung adenocarcinomas and squamous cell carcinomas. Nat Genet 2016;48:607-16.

9. Ning P, $\mathrm{Wu} Z, \mathrm{Hu} A$, et al. Integrated genomic analyses of lung squamous cell carcinoma for identification of a possible competitive endogenous RNA network by means of TCGA datasets. PeerJ 2018;6:e4254.

10. Cancer Genome Atlas Research N. Comprehensive genomic characterization of squamous cell lung cancers. Nature 2012;489:519-25.

11. Cancer Genome Atlas Research N. Comprehensive molecular profiling of lung adenocarcinoma. Nature 2014;511:543-50.

12. Mercer TR, Dinger ME, Mattick JS. Long noncoding RNAs: insights into functions. Nat Rev Genet 2009;10:155-9.

13. Fang Y, Fullwood MJ. Roles, Functions, and Mechanisms of Long Non-coding RNAs in Cancer. Genomics Proteomics Bioinformatics 2016;14:42-54.

14. Arun G, Diermeier SD, Spector DL. Therapeutic Targeting of Long Non-Coding RNAs in Cancer. Trends Mol Med 2018;24:257-77.

15. Chen L, Dzakah EE, Shan G. Targetable long non-coding RNAs in cancer treatments. Cancer Lett 2018;418:119-24.

16. Wang L, Yu Z, Sun S, et al. Long non-coding RNAs: potential molecular biomarkers for gliomas diagnosis and 
prognosis. Rev Neurosci 2017;28:375-80.

17. Deng H, Wang JM, Li M, et al. Long non-coding RNAs: New biomarkers for prognosis and diagnosis of colon cancer. Tumour Biol 2017;39:1010428317706332.

18. Salmena L, Poliseno L, Tay Y, et al. A ceRNA hypothesis: the Rosetta Stone of a hidden RNA language? Cell 2011;146:353-8.

19. Conte F, Fiscon G, Chiara M, et al. Role of the long noncoding RNA PVT1 in the dysregulation of the ceRNAceRNA network in human breast cancer. PLoS One 2017;12:e0171661.

20. Fan CN, Ma L, Liu N. Systematic analysis of lncRNAmiRNA-mRNA competing endogenous RNA network identifies four-lncRNA signature as a prognostic biomarker for breast cancer. J Transl Med 2018;16:264.

21. Liu $\mathrm{H}$, Zhang $\mathrm{Z}, \mathrm{Wu} \mathrm{N}$, et al. Integrative Analysis of Dysregulated lncRNA-Associated ceRNA Network Reveals Functional lncRNAs in Gastric Cancer. Genes (Basel) 2018;9. pii: E303.

22. Lu M, Xu X, Xi B, et al. Molecular Network-Based Identification of Competing Endogenous RNAs in Thyroid Carcinoma. Genes (Basel) 2018;9. pii: E44.

23. Cao Y, Wang P, Ning S, et al. Identification of prognostic biomarkers in glioblastoma using a long non-coding RNA-mediated, competitive endogenous RNA network. Oncotarget 2016;7:41737-47.

24. Du Z, Sun T, Hacisuleyman E, et al. Integrative analyses reveal a long noncoding RNA-mediated sponge regulatory network in prostate cancer. Nat Commun 2016;7:10982.

25. Zhou M, Wang X, Shi H, et al. Characterization of long non-coding RNA-associated ceRNA network to reveal potential prognostic lncRNA biomarkers in human ovarian cancer. Oncotarget 2016;7:12598-611.

26. Sui J, Xu SY, Han J, et al. Integrated analysis of competing endogenous RNA network revealing lncRNAs as potential prognostic biomarkers in human lung squamous cell carcinoma. Oncotarget 2017;8:65997-6018.

27. Tang RX, Chen WJ, He RQ, et al. Identification of a RNA-Seq based prognostic signature with five lncRNAs for lung squamous cell carcinoma. Oncotarget 2017;8:50761-73.

28. Langfelder P, Horvath S. WGCNA: an R package for weighted correlation network analysis. BMC Bioinformatics 2008;9:559.

29. Ding M, Li F, Wang B, et al. A comprehensive analysis of WGCNA and serum metabolomics manifests the lung cancer-associated disordered glucose metabolism. J Cell Biochem 2019;120:10855-63.
30. Di Y, Chen D, Yu W, et al. Bladder cancer stage-associated hub genes revealed by WGCNA co-expression network analysis. Hereditas 2019;156:7.

31. Zhai X, Xue Q, Liu Q, et al. Colon cancer recurrenceassociated genes revealed by WGCNA coexpression network analysis. Mol Med Rep 2017;16:6499-505.

32. Robinson MD, McCarthy DJ, Smyth GK. edgeR: a Bioconductor package for differential expression analysis of digital gene expression data. Bioinformatics 2010;26:139-40.

33. Szklarczyk D, Franceschini A, Wyder S, et al. STRING v10: protein-protein interaction networks, integrated over the tree of life. Nucleic Acids Res 2015;43:D447-52.

34. Shannon P, Markiel A, Ozier O, et al. Cytoscape: a software environment for integrated models of biomolecular interaction networks. Genome Res 2003;13:2498-504.

35. Bader GD, Hogue CW. An automated method for finding molecular complexes in large protein interaction networks. BMC Bioinformatics 2003;4:2.

36. Yu G, Wang LG, Han Y, et al. clusterProfiler: an R package for comparing biological themes among gene clusters. OMICS 2012;16:284-7.

37. Tang Y, Li M, Wang J, et al. CytoNCA: a cytoscape plugin for centrality analysis and evaluation of protein interaction networks. Biosystems 2015;127:67-72.

38. Tang Z, Li C, Kang B, et al. GEPIA: a web server for cancer and normal gene expression profiling and interactive analyses. Nucleic Acids Res 2017;45:W98-W102.

39. Chandrashekar DS, Bashel B, Balasubramanya SAH, et al. UALCAN: A Portal for Facilitating Tumor Subgroup Gene Expression and Survival Analyses. Neoplasia 2017;19:649-58.

40. Li L, Chen K, Xiang Y, et al. New development in studies of formyl-peptide receptors: critical roles in host defense. J Leukoc Biol 2016;99:425-35.

41. Hou XL, Ji CD, Tang J, et al. FPR2 promotes invasion and metastasis of gastric cancer cells and predicts the prognosis of patients. Sci Rep 2017;7:3153.

42. Xiang Y, Yao X, Chen K, et al. The G-protein coupled chemoattractant receptor FPR2 promotes malignant phenotype of human colon cancer cells. Am J Cancer Res 2016;6:2599-610.

43. Hossain MN, Sakemura R, Fujii M, et al. G-protein gamma subunit GNG11 strongly regulates cellular senescence. Biochem Biophys Res Commun 2006;351:645-50.

44. Takauji Y, Kudo I, En A, et al. GNG11 (G-protein 
subunit gamma 11) suppresses cell growth with induction of reactive oxygen species and abnormal nuclear morphology in human SUSM-1 cells. Biochem Cell Biol 2017;95:517-23.

45. Liu J, Sun X, Zhu H, et al. Long noncoding RNA POU6F2-AS2 is associated with oesophageal squamous cell carcinoma. J Biochem 2016;160:195-204.

46. Huang H, Wang Y, Li Q, et al. miR-140-3p functions as a tumor suppressor in squamous cell lung cancer by regulating BRD9. Cancer Lett 2019;446:81-9.

47. Zhao Z, Liu W, Li J. miR-140-5p inhibits cell proliferation and invasion in colorectal carcinoma by targeting SOX4.

Oncol Lett 2019;17:2215-20.

Cite this article as: $\mathrm{Hu} \mathrm{J}, \mathrm{Xu} \mathrm{L}$, Shou T, Chen Q. Systematic analysis identifies three-lncRNA signature as a potentially prognostic biomarker for lung squamous cell carcinoma using bioinformatics strategy. Transl Lung Cancer Res 2019;8(5):614635. doi: 10.21037/tlcr.2019.09.13
48. Zhou Y, Wang B, Wang Y, et al. miR-140-3p inhibits breast cancer proliferation and migration by directly regulating the expression of tripartite motif 28 . Oncol Lett 2019;17:3835-41.

49. Zou MX, Huang W, Wang XB, et al. Identification of miR-140-3p as a marker associated with poor prognosis in spinal chordoma. Int J Clin Exp Pathol 2014;7:4877-85.

50. Bi DP, Yin CH, Zhang XY, et al. MiR-183 functions as an oncogene by targeting ABCA1 in colon cancer. Oncol Rep 2016;35:2873-9.

51. Zhang L, Quan H, Wang S, et al. MiR-183 promotes growth of non-small cell lung cancer cells through FoxO1 inhibition. Tumour Biol 2015;36:8121-6. 


\section{Supplementary}

CACNA2D3-AS1

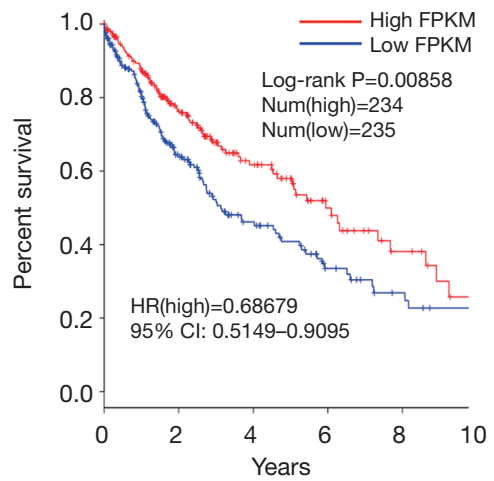

LNX1-AS1

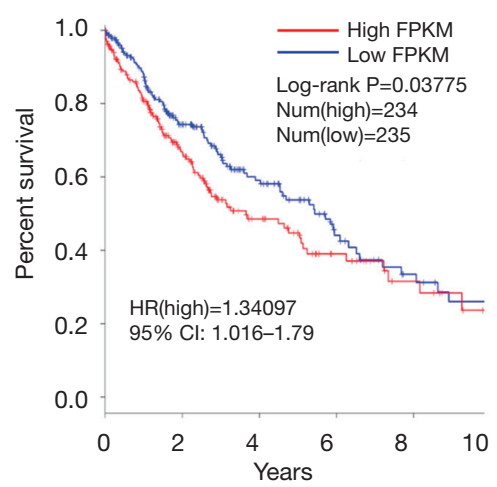

POU6F2-AS2

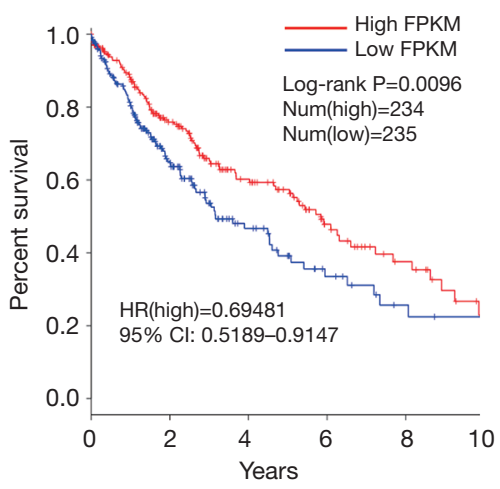

KCNQ5-IT1

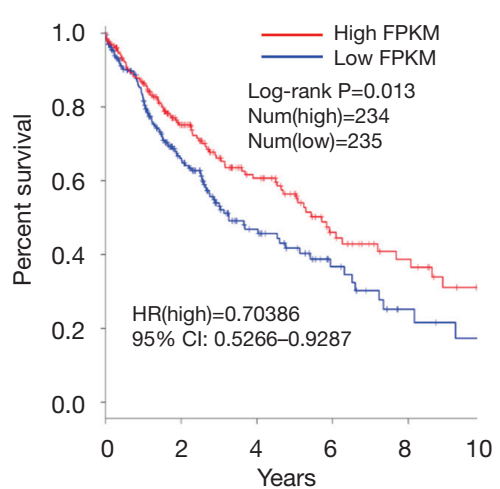

TाTY16

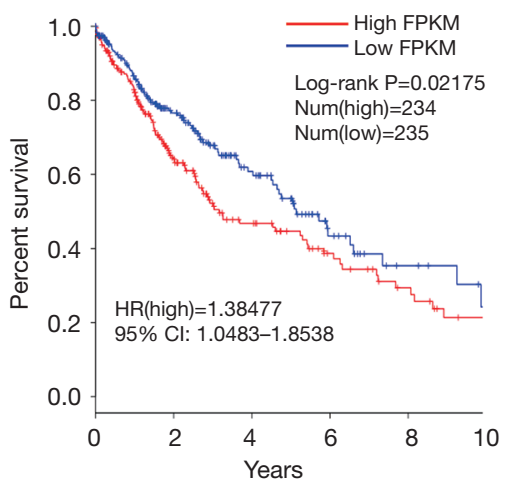

AL391152.1

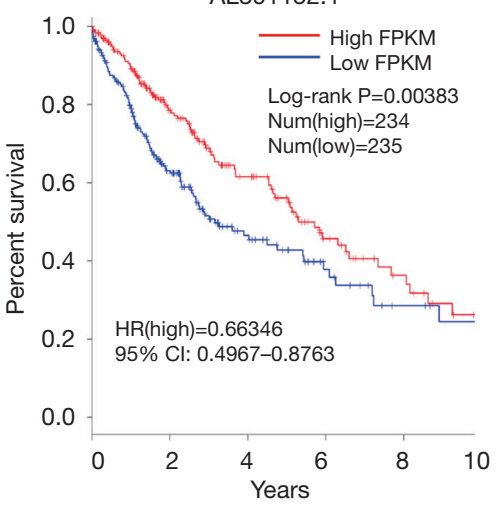

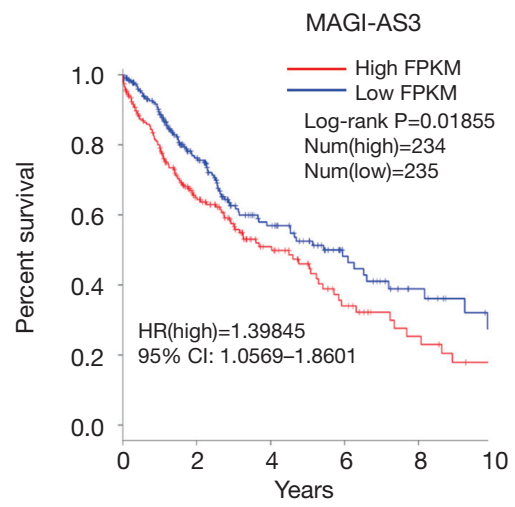

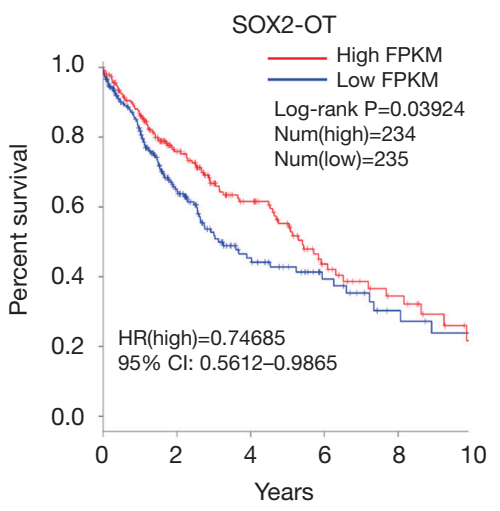

Figure S1 The relationships of lncRNAs and overall survival of lung squamous cell carcinoma (LUSC) patients. 